\title{
In-vitro Investigation of Polymeric Lipid Hybrid Nanoparticles (Plhns) Of a Chemotherapeutic Drug for the Treatment of Glioblastoma Multiforme
}

\author{
Sankha Bhattacharya ${ }^{1^{*}}$ \\ ${ }^{1}$ Department of Pharmaceutics, School of Pharmacy \& Technology Management, SVKM'S NMIMS \\ Deemed-to-be University, Shirpur, Maharashtra 425405, India
}

*Corresponding author:

Name: Dr Sankha Bhattacharya

Designation: Associate professor

Department: Pharmaceutics

Affiliation

School of Pharmacy \& Technology Management, SVKM'S NMIMS Deemed-tobe University, Shirpur, Maharashtra 425405, India

Address:

School of Pharmacy \& Technology Management Shirpur, SVKM'S NMIMS

Deemed-to-be University, Shirpur, Maharashtra 425405, India

Mobile: +917878777207

Email ID: sankhabhatt@gmail.com

ORCID ID: 0000-0002-0771-9582 


\section{$\underline{\text { Graphicl Abstract }}$}

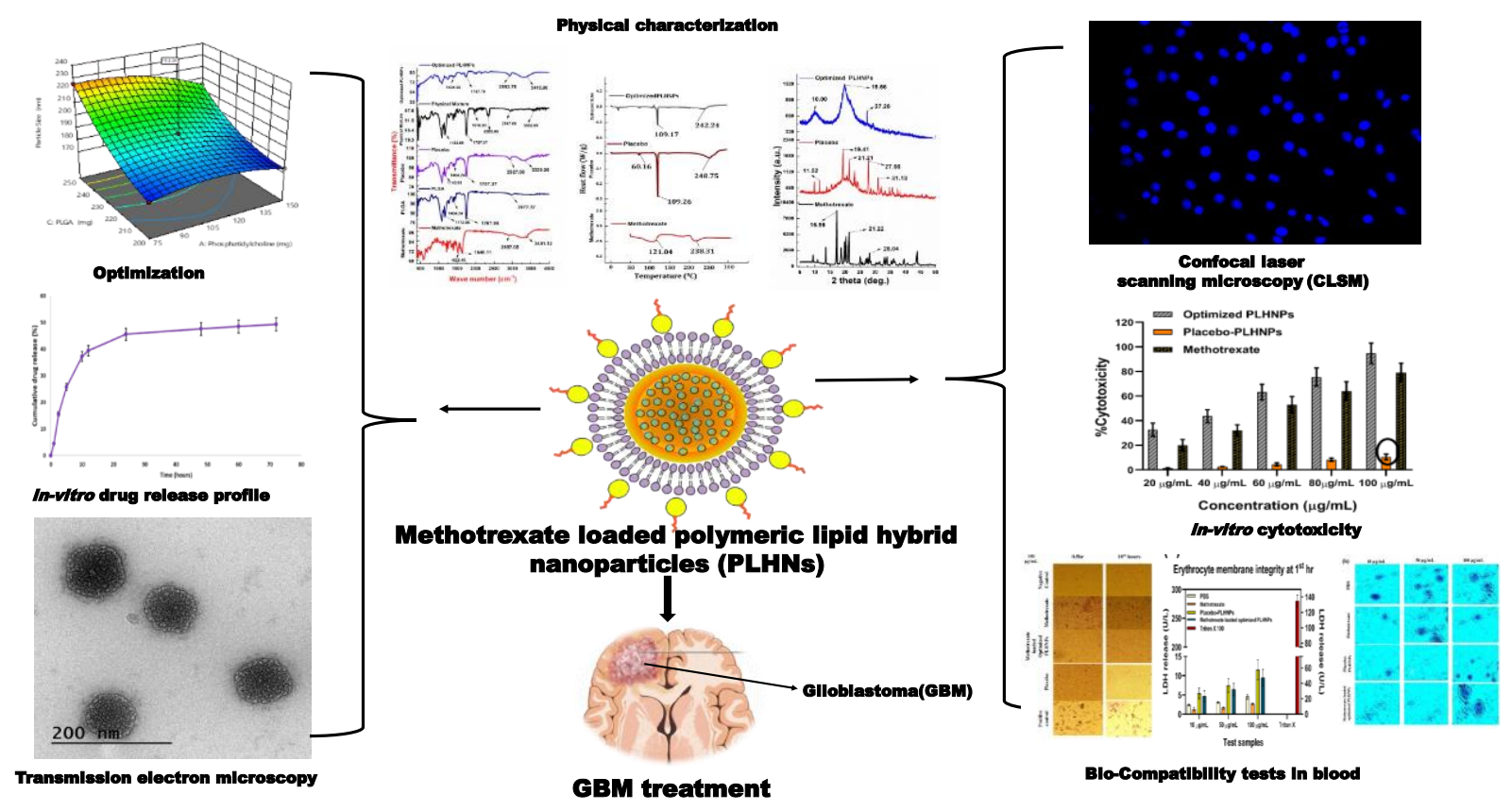

Methotrexate loaded polymeric lipid hybrid nanoparticles (PLHNs) 


\title{
In-vitro investigation of polymeric lipid hybrid nanoparticles (PLHNs) of a chemotherapeutic drug for the treatment of glioblastoma multiforme
}

\begin{abstract}
:
PHLNs (polymeric lipid hybrid nanoparticles) are core-shell nanoparticle structures made up of polymer cores and lipid shells that have properties similar to both polymeric nanoparticles and liposomes. Methotrexate (MTX) loaded PLHNPs containing tween 80, phosphatidylcholine, poly D, L-lactic-co-glycolic acid (PLGA) \& glyceryl tripalmitate prepared using solvent injection \& homogenization method for glioblastoma treatment option. The MTX loaded PLHNPs optimized by Box-Behnken design to minimize particle size, higher entrapment efficacy, and maximize MTX concentration in the brain at $4 \mathrm{~h}$. The particle size, entrapment efficacy, concentration of drug in brain at $4 \mathrm{~h}$, zeta potential and $\mathrm{AUC}_{(\mathrm{Brain}) /} \mathrm{AUC}_{\text {(Plasma) }}$ ratio were in the range of 173.51233.37nm, 70.56-86.34\%, 6.38-12.38 $\mu \mathrm{g} / \mathrm{mL}, 25.78-36.31 \mathrm{mV} \& 1.02-5.32$. in-vitro drug release studies, cellular internalization of optimized formulation against U-87 MG shows good anticancer effects.
\end{abstract}

\section{Keywords:}

Cytotoxicity; Glioblastoma multiform; Lactate dehydrogenase (LDH); Poly D- L-lactic-coglycolic acid (PLGA); U-87 MG glioma cell lines 


\section{Abbreviations:}

\%EE: Percentage entrapment efficiency

ANOVA: Analysis of variance

AUC: Area under the curve

BBB: blood-brain barrier

DAPI: 4',6-diamidino-2-phenylindole

DCM: Dichloromethane

DLS: Dynamic light scattering

DSC: Differential scanning calorimetry

GBM: Glioblastoma multiform

KBr: Potassium bromide

LDH: Lactate dehydrogenase

MDA: Malondialdehyde

MTT: 3-(4,5-dimethyl thiazol-2-yl)-2,5-diphenyltetrazolium bromide

MTX: Methotrexate

PBS: Phosphate buffered saline

PLGA: Poly(lactic-co-glycolic acid)

PLHNPs: Polymeric-lipid hybrid nanoparticles

PVA: Polyvinyl alcohol

TEM: Transmission electron microscopy 


\section{In-vitro investigation of polymeric lipid hybrid nanoparticles (PLHNs) of a chemotherapeutic drug for the treatment of glioblastoma multiforme}

\section{Introduction:}

Nanotechnology is playing a pivotal role while fighting against cancer. Many drugs, genes, and other macromolecules can be used to encapsulate or scaffold within nanoparticles[1, 2]. This immuno inert nanocarrier system triggers targeted delivery at the site of action, resulting in increased therapeutic activity. Polymeric and lipid nanoparticles are commonly used in such nanocarrier systems, and these nanocarrier systems are tailored using a variety of alteration techniques to achieve higher therapeutic efficacy[3]. This, in turn, aids in improving nanocarrier bio-stability and target specificity. Long-circulating chemotherapeutic agents, for example, can be encapsulated within modified nanocarrier systems with active targeting ligands like genes, protein, hormone, folic acid, and so on to activate the enhanced permeability and retention (EPR) effect and reticuloendothelial opsonization[4]. When it comes to polymeric nanoparticles, these nanocarrier systems have good structural integrity and stability during storage; additionally, changes in preparative methods could improve their functionality and target specificity[5]. PLGA, PEG, and chitosan have been shown to reduce nano-specific interaction and improve the biodegradability and biocompatibility of polymeric nanoparticles in several studies[6]. However, because lipid nanoparticles, such as Liposomes and Nosomes, are nearly identical to biological membranes, they appear to be superior to polymeric nanoparticles in terms of biocompatibility and target specificity[7]. Furthermore, both natural and synthetic phospholipids can be used to 
make lipid nanoparticles. Contrary to popular belief, liposomes are cleared by the RES system due to their lack of structural integrity and leaky nature, resulting in poor bioavailability[8]. Recent research suggests that lipid-PEG liposomes can be combined to create stable nanoparticles. A unique and new generation nano vehicle called polymeric lipid hybrid nanoparticles (PLHNs) was recently developed to address the limitations of polymeric and lipidic nanoparticles[9]. These hybrid nanoparticles combine the benefits of polymeric and liposomal nanoparticles, as well as the possibility of a stealth coating, to extend in-vitro circulation time and improve biocompatibility and stability $[9,10]$. Recent research on the preparation of PLHNs has reduced two-step synthesis to one, resulting in increased development throughput. To overcome the difficulties of lipid-based nanoparticles, more research needs to emphasize on polymeric-lipid hydride nanoparticular framework, which could provide strategic advantages by combining core polymeric materials and lipid shells' ridged structural integrity[11]. The entire drug payload can be retained in these robust polymeric-lipid hydride nanoparticles, and the outer hydrophobic lipid coating can prevent water from entering the central polymer to channelize diffusion[12].

As per Jiayi Gu et al. (2020) studies, Astaxanthin encapsulated lipid-polymer hybrid nanoparticles has a greater effect against cisplatin induced toxicity[13]. As per Salam Massadeh et al. (2020) research, Anastrozole was encapsulated by a polyethylene glycolated (PEGylated) polymer-lipid hybrid nanoparticulate device using a direct emulsification solvent evaporation process[14]. It has a better encapsulation efficacy and superior anticancer effects against MCF-7 cell lines. Similarly, as per Xiangzhao Ai et al. (2021) studies, in mice cartilage injury, collagen-peptide shell and PLGA core lipidpolymer hybrid nanoparticles may be very useful as a control drug delivery[15].

According to multiple research findings and patents, polymeric-lipid hybrid nanoparticles (PLHNPs) could have a sustained release effect on the encapsulated compound[16, 17]. Adsorption of an appropriate stabiliser or surfactant on the outer layer of the core-shell may also improve nanoparticles permeability through the blood-brain barrier (BBB) [18].Recent research findings also indicate that during treatment, extracellular serum glycoproteins, such as vitronectin, can adsorb to the surface of PLHNPs, enhancing anticancer activity. 
PLGA (Poly (D, L-lactide-coglycolide)) was used to improve the drug profile in this study[19]. Furthermore, PLGA-encapsulated nanoparticles protect medications from degradation and aid absorptive transcytosis into the endothelial cortex[20]. The reticuloendothelial system is restrained from PLGA solid lipid nanoparticles below 200nm (RES)[21]. As a result, the primary aim of this study was to make drug-loaded polymeric-lipid hybrid nanoparticles (PLHNPs) for better stability and rigidity of the formulation[22].

It's often challenging to get the correct medicament for glioblastoma or glioma (GBM) treatment. Methotrexate (MTX) was considered after a thorough analysis of the literature. Methotrexate (MTX) is a drug used to combat cancers such as non-small cell lung cancer, breast cancer, and ovarian cancer[23]. However, only a few experiments have been triggered to treat GBM with PLGA solid lipid nanoparticles filled with methotrexate (MTX)[24]. For different cancer therapies, commercial Methotrexate (MTX) tablets are available: Folitrax ${ }^{\circledR}$, Mext ${ }^{\circledR}$, Mexate ${ }^{\circledR}$, Biotrexate ${ }^{\circledR}[25]$, Oncotrex ${ }^{\circledR}$, Merex ${ }^{\circledR}$, Methocel ${ }^{\circledR}$. However, most tablets are cytotoxic, lack target specificity, and degrade more quickly, resulting in poor bioavailability. Polymeric solid lipid drug distribution through the intravenous (IV) route would be the best way to solve both of these issues, as polymeric lipid particles (>200nm) will penetrate the systematic circulation and in PLGA matrix lipidic shell coating over MTX will shield it from macrophagic absorption and reticuloendothelial opsonization. As a consequence, MTX could enter the brain and be processed by endothelial cells[26].

As compared to tablets, the key benefits of PLHNPs are increased bioavailability, sustainedrelease properties, and less side effects.Therefore, this investigation aimed to emphasize formulation and in-vitro investigation of of MTX loaded PLHNPs using Box-Behnken design for potential GBM treatment option. To obtain the prime objective of this study a single step synthesis was performed where MTX loaded PLHNPs were synthesised using simple solvent injection method. The polymeric-lipid hybrid nanoparticles (PLHNPs) comprising of PLGA in core and glyceryl tripalmitate \& phosphatidylcholine in shell. To stabilize and maintain rigidity, polyvinyl alcohol (PVA) was added into PLHNPs formulation (Fig.1) to strengthen and maintain rigidity. 
The research also sought to determine the physical compatibility (FTIR, DSC, XRD), particle size, zeta potential, entrapment efficiency, morphology (TEM), in-vitro drug release, brain uptake potential \& pharmacokinetics studies, anticancer activity against U-87 MG glioma cells, cellular uptake, biocompatibility (Lactate dehydrogenase assay, platelet aggregation, haemolysis) studies of synthesised PLHNPs.

\section{Materials and methods:}

\subsection{Materials}

The Drug; Methotrexate, was a gift sample from Neon Laboratories Ltd, Mumbai. The poly D, L-lactic-co-glycolic acid (PLGA) 75:25 was provided by Nomisma Healthcare, Gujarat. The tween 80, phosphatidylcholine, glyceryl tripalmitate, PVA were purchased from Sigma-Aldrich, Bengaluru. Analytical grade methanol, ethanol, and other organic chemicals were purchased from Sisco Research Laboratories Pvt. Ltd, Mumbai. The DMEM (Dulbecco's Modified Eagle Medium), human serum albumin (HAS), 3-(4,5-Dimethylthiazol-2-yl)-2,5-Diphenyltetrazolium Bromide (MTT), 4',6-diamidino-2-phenylindole (DAPI) was procured from Sigma-Aldrich, Bengaluru. The Lactate Dehydrogenase Assay Kit (Colorimetric) was procured from Abcam laboratories, USA. Rest all the chemicals used in various experiments of this research were of analytical grades.

\subsection{Preparation of PLGA solid lipid nanoparticles:}

Methotrexate-containing PLGA polymeric-lipid hybrid nanoparticles (PLHNPs) were prepared using solvent injection system with necessary modifications using methanol as organic solvent[27]. The aqueous phase comprising $2.5 \mathrm{mg}$ of Methotrexate dissolved in $0.75 \%$ tween 80 , phosphatidylcholine, $1.5 \% \mathrm{w} / \mathrm{v}$ PVA(Stabilizer) and PLGA was dissolved in the hydroalcoholic mixture; methanol (25\%) and double distilled water (75\%). The lipid step consisted of various glyceryl tripalmitate quantities (about 99\%). The aqueous and lipid process was warmed at $80^{\circ} \mathrm{C}$ above the lipid melting point. Using a hydrodynamic needle, in the previously heated water phase, the molten lipid phase eventually fell. When inserting, sustain 15 minutes of homogenization speed utilising Ultra Turrax T 25 Digital Homogenizer System. 
Though homogenising, organic residue vaporisation mechanism was left available. The subsequent liquid was prob-sonicated for $15 \mathrm{~min}$ after homogenization (Sonifier® SFX550 Cell Disruptors and Homogenizers, Thomas Scientific, USA). The resulting liquid was centrifuged at 20,000 rpm at $4 \mathrm{oC}$ for 30 minutes (Refrigerated Centrifuge 5702R, Thomas Scientific, USA). During centrifugation, a white pellet layer was clear at the centrifugation tube rim. The supernatant was analysed for drug quality tests and three times washed the pellet layer using HPLC grade water. Delsa Nano C (Beckman Coulter, U.S.A.) calculated particle size and zeta potential of nanoparticles prepared. In Free Zone ${ }^{\circledR}$ Triad® Benchtop Freeze Dryers (Thomas Science, USA), the nanoparticles obtained were further lyophilized considering $2.5 \% \mathrm{w} / \mathrm{w} \mathrm{d}-$ trehalose as cryoprotective.

\subsection{Optimization using Box-Behnken design:}

Preliminary screening's key objective was to evaluate the various factors influencing particle size, zeta potential, and trap efficacy of prepared nanoparticles. The most significant factors were the concentration of phosphatidylcholine, glyceryl tripalmitate, and PLGA. The design of BoxBehnken[28]; where quadratic reaction surface design system RSM was introduced, the influence of three factors (phosphatidylcholine, glyceryl tripalmitate, and PLGA) on the dependent variables, i.e. particle size(Y1), zeta potential(Y2) and entrapment efficacy(Y3), the concentration of drug in the brain at $4 \mathrm{~h}$ interval(Y4), AUC(Brain)/AUC(PPC) (Y5) was evaluated. In total, thirteen experimental runs were conducted and nanoparticles were prepared randomly to avoid possible unreliable outcomes. Table 1 outlined the experimental design data. Model-fitting analysis was performed using programme design-expert.

\section{Evaluation and characterization of solid lipid nanoparticles:}

\subsection{Particle size analysis and zeta potential determination:}

The average particle size and zeta potential were determined using Delsa Nano C (Beckman Coulter, USA)[29]. Where nanoparticles were suspended in HPLC water and analysed through triplicate photon correlation spectroscopy[30]. Measurement of particle size in Delsa Nano C at 
a scattering angle of $90^{\circ}$ at $25^{\circ} \mathrm{C}$ for 120 cycles. For zeta-potential measurement, $0.5 \mathrm{~mL}$ of the sample was put in the electrophoretic flow cell and measurement was taken for 80 cycles.

\subsection{Entrapment efficiency (EE):}

The prepared nanosuspension was initially centrifuged at $4^{\circ} \mathrm{C}$ for $30 \mathrm{~min}$ at $15,000 \mathrm{rpm}$. During centrifugation, an identical formation of pellets can be seen within the centrifuge tubbings at the rim. By means of decantation the supernatant was obtained and analysed using the Perkin Elmer LAMBDA XLS Spectrometer in UV Visible spectroscopic method in triplicate at $244 \mathrm{~nm}$. The blank was taken as the supernatant nanoparticular which was prepared without any drug[31]. To obtain regression equation $\mathrm{y}=0.056 \mathrm{x}+0.18$ (co-efficient of correlation $\mathrm{R} 2=0.0997$ ), the normal calibration curve of Methotrexate between absorbance (y) and concentration (x) was plotted. The following equation was used to calculate percentage drug entrapment efficacy:

Drug entrapment efficiency $($ EE \% $)=\frac{\text { Total drug content }- \text { Amount of free drug }}{\text { Total drug content }} \times 100$

\subsection{In-vitro drug release study of the optimized formulation:}

Studies of the in vitro drug release were performed in the Spectra Por S/P 2 Dialysis Membrane Trial tube (10,000-12,000Dalton 50mm)[32]. The dialysis tubes were preloaded with nanoparticles that must have $2.5 \mathrm{mg}$ of methotrexate and $2 \mathrm{~mL}$ of phosphate buffer solution before being clamped with the USP Type II dissolution apparatus ( $\mathrm{pH}$ 7.4). The paddle was rotated at $100 \mathrm{rpm}$, and the dissolution medium was known to be a $500 \mathrm{~mL}$ (pH7.4) phosphate buffer solution while retaining $37 \pm 2.5^{\circ} \mathrm{C}$. The aliquoted sample quantity $(5 \mathrm{~mL})$ was removed from the dissolution medium during perforation of the examination, and the same amount of freshly formulated buffer solution was added to the dissolution medium. The material was analysed spectrophotometrically at $244 \mathrm{~nm}$ in triplicate. In addition, triplicate breakup experiments were also carried out, and the results were expressed in terms of percentage of opioid release \pm standard deviation. For dissolution purposes the $\mathrm{pH} 7.4$ phosphate buffer solution was used to maintain the sink condition. 


\subsection{In vivo studies:}

Before beginning the experiment, the animals were properly acclimatised to laboratory environments, and every precaution was taken to avoid any discomfort. The animals were given an adequate diet and free access to water before being placed in a 24-h light/dark cycle[33]. To perform in vitro experiments, Wister rats (250-300 g) were acquired. The Committee for the Monitoring and Supervision of Animal Experiments (CPCSEA) of the Ministry of Environment and Forests (Animal Welfare Division) closely adopted the Government of India guidelines [DPL/1410/c/11/2020/CPCSEANo.458] before any animal experiments. Deshpande Laboratories, Pvt. Ltd., Bhopal, India, received the animaland conducted the tests.

\subsection{Fourier -transform infrared (FTIR) spectroscopy:}

To clarify Methotrexate's potential interaction with different excipients, Fourier-transform infrared (FTIR) spectroscopy studies were performed[34]. The FTIR spectroscopy was estimated from 400-4000 $\mathrm{cm}^{-1}$. The FTIR spectra of lyophilized drug-loaded optimized polymeric nanoparticles, the physical mixture of drug and other excipients, placebo nanoparticles, and pure methotrexate drug were reported. The experiment was conducted in Frontier NIR Spectrometer (Perkin Elmer, USA) using the $\mathrm{NaBr}$ pellet method to observe chemical interactions inside the formulation between Methotrexate and other carrier composition.

\subsection{Differential scanning calorimetry (DSC):}

Using a Differential Scanning Calorimeter-800, the DSC thermograph of Methotrexate, optimised formulation with Methotrexate and palcebo formulation without drug was measured (PerkinElmer, USA)[35]. DSC experiments can establish the formulation's physical nature and patterns of drug and polymer behaviour. To perform the DSC thermogram analysis, each individual sample was mounted in crimped aluminium and heat flow ranged from $25-400^{\circ} \mathrm{C}$ to $15^{\circ} \mathrm{C} / \mathrm{min}$. Nitrogen gas circulated at $150 \mathrm{~mL} / \mathrm{min}$ flow rate to maintain inert condition. Origin Pro 8.5 V Software analyzed the extracted CSV file and analysed data. 


\subsection{Powder-X Ray -diffraction study:}

To recognise the crystal nature of the compound, X-Ray Diffraction tests were conducted for pure drug, drug-optimized formulation and optimised formulation with ARLTM EQUINOX $100 \mathrm{X}$ ray Diffractometer[36]. This experiment used a high-intensity X-Ray source. The diffraction pattern was reported at $2 \Theta$ and between $30^{\circ} \mathrm{C}$ to $80^{\circ} \mathrm{C}$ with an incremental degree of $0.03^{\circ}$ and 3 seconds per phase.

\subsection{Transmission Electron microscopy:}

To investigate the surface morphology and particle size of prepared polymeric nanoparticles, the optimised batch was diluted with HPLC grade water and tested at $120 \mathrm{kV}$ under transmission electron microscopy (JEM-ARM300F GRAND ARM Atomic Resolution Electron Microscope)[37]. The samples were Freshly prepared using 2\% phosphotungstic acid (pH 7.0) and put on a carbon-coated grid (300 mesh).

\subsection{Determination of brain uptake potential:}

The concentration of drugs in the brain and 4th hour AUC brain/AUC plasma administration must be measured for optimisation purposes[38]. These two were known as response variables in formulation optimization. For brain absorption trials of pure drug, optimised formulation, placebo, control (normal saline), male Wister rats were divided into four groups of 3 animals each group. Before experimenting, the animals were kept for fasting. Water feed was kept accurate; however, pure drug (MTX), test formulation, was administered during intravenous (IV) testing. Blood samples $(0.5 \mathrm{~mL})$ were obtained in heparin-containing tubes. After extracting blood samples in various time intervals, i.e., 30, 60, 120, and $240 \mathrm{~min}$, the samples were centrifuged for $20 \mathrm{~min}$ at $13000 \mathrm{rpm}$, and plasma samples were collected and processed at $-48^{\circ} \mathrm{C}$. Samples were for analytical purposes.

By decapitation, animals were executed after extracting blood samples in the presence of anaesthesia. Carefully removed brains and washed blood with bottling sheets. Brain tissues were 
homogenised with a $0.01 \mathrm{M}$ phosphate buffer ( $\mathrm{pH} 7.4$ ) in tissue homogenizer (f NS-52 Tissue Homogenizer, Microtec, Japan) for $15 \mathrm{~min}$ and centrifuged at $13000 \mathrm{rpm}$. The resultant supernatant was deposited at $-30^{\circ} \mathrm{C}$.

The amount of drug in brain samples was measured using HPLC. During the trial, $100 \mu \mathrm{g} / \mathrm{mL}$ methotrexate $(20 \mu \mathrm{L})$ was taken and dissolved in $2 \mathrm{M}$ sodium hydroxide solution and mixed properly. A plasma/brain sample of $300 \mu \mathrm{L}$ poured the mixture. The mixture was thoroughly vortexed, applying ethyl acetate, and 30 minutes shaking at $15^{\circ} \mathrm{C}$. The process was repeated three times, the constituents' supernatant dried and reconstituted $400 \mu \mathrm{L}$ mobile phase. To quantify the volume of drug present in plasma and brain, correctly tested HPLC methods are used. The samples were properly screened using a $0.22 \mu \mathrm{m}$ syringe filter and the requisite sample quantity was injected into the well-equipped Waters Alliance 2695 HPLC System. Methotrexate HPLC was determined in $303 \mathrm{~nm}$ with a flow rate of $1.5 \mathrm{~mL} / \mathrm{min}$. Mobile phase consisted of buffer and acetonitrile (98:2). Dissolving $27.22 \mathrm{~g}$ potassium dihydrogen orthophosphate in $1000 \mathrm{~mL}$ purified water prepared the Buffer solution. During the HPLC method, the $\mathrm{pH}$ with $\mathrm{NaOH}$ solution was changed to $6.0 \pm 0.05$ and the resulting mixture was filtered using a $0.2 \mu$ nylon membrane filter. The propensity for brain and plasma medication was measured by testing the drug in brain and plasma. Also, AUC brain/AUC plasma was measured using the trapezoidal method.

\subsection{Cytotoxicity studies against U-87 MG glioma cells:}

Using MTT assay in vitro Methotrexate cytotoxicity, drug-loaded optimised formulation, placebo was calculated in U-87 MG cell lines[39]. The cells were seeded onto 96-Well Cell Culture Plates (Thermofisher Science, USA) at 1x104 cells/well in Dulbecco's Updated Eagle's Medium (DMEM) and incubated for 36 hours in a $\% \mathrm{CO}_{2}$ incubator while performing the experiment. The cells were subjected to various research conditions when incubating at $37^{\circ} \mathrm{C}$ in a $5 \% \mathrm{CO}_{2}$ environment for 72 hours. In addition, $40 \mu \mathrm{L}$ of 3-(4,5-dimethylthiazol-2-yl)-2,5-diphenyl tetrazolium bromide (MTT) solution was added to the culture medium and incubated for 8 hours at $37^{\circ} \mathrm{C}$. Insoluble formazan crystals form during the reaction phase, which are proportional to the number of viable cells. These insoluble formazan crystals were dissolved in $100 \mathrm{~L}$ dimethyl 
sulfoxide (DMSO), the plates were vigorously agitated for 20 minutes, and absorption at $580 \mathrm{~nm}$ was determined using Multi-Mode and Absorbance Readers (Bio Tek, USA). The following equation was used to quantify the percentage of cytotoxicity:

$$
\% \text { Cytotoxicity }=100-\frac{\text { Absorbance of test }}{\text { Absorbance of control }} \times 100
$$

\subsection{Cellular uptake:}

The U-87 MG glioma cells were propagated in a $35 \mathrm{~mm}$ petri dish at $1 \times 104$ cells/cover glass cells, in the presence of Dulbecco's Modified Eagle Medium (DMEM). At $37^{\circ} \mathrm{C}$ and further exposed with refined formulation (100 $\mu \mathrm{L} /$ cover glass) in the humidified $\mathrm{CO} 2(5 \%)$ incubator. After 4 hours, cells were correctly washed three times using a 7.4pH phosphate buffer solution, and later the cells were fixed with paraformaldehyde for $40 \mathrm{~min}$. The cells were further washed with phosphate buffer solution three times after $40 \mathrm{~min}$. Furthermore, cells were stained for $20 \mathrm{~min}$ using $0.1 \mu \mathrm{g} / \mathrm{mL}$ of DAPI reagent. The developed monolayer was further washed with phosphate buffer solution inside the cover glass and the slides were examined under a confocal laser microscope (Anamatrix Laser Scanning Microscope).

\subsection{Evaluation of haemolysis:}

On the basis of a previous publications, the drug, optimised formulation, and placebo were analysed. Since the drug can be absorbed from the blood capillaries and, as a result, possibilities of haemolyse blood cells after ingestion is high. Therfoer, a haemolysis analysis is needed to support the product's biocompatibility. Hemocompatibility tests will also provide us an understanding about how the drug, final product, and placebo will respond to blood[40].

Blood was obtained from the nearest blood bank to conduct haemolysis tests[41]. The blood samples $(2.5 \mathrm{~mL})$ were centrifuged for 15 minutes at $1345 \mathrm{xg}$ at room temperature. The graduated centrifuge tubes used in this experiment were previously sterile. Micropipettes were used to scrape the plasma coating during centrifugation. The developed erythrocyte pellets in the bottom of the graduated centrifuge tubes were diluted and suspended further using normal saline solution. At room temperature, the suspended part was centrifuged against $1345 \mathrm{xg}$ for 15 minutes. Centrifugation was used three times to finish the washing operation. The erythrocyte suspension 
was further diluted with $10 \mathrm{~mL}$ saline water, and then 10,50 , and $100 \mathrm{~g} / \mathrm{mL}$ of pure medication, optimised dosage, and placebo were each combined with $2 \mathrm{~mL}$ of erythrocyte suspension in sterile tubes separately. The positive regulated examination, which fully lyzed erythrocytes, was rendered by dissolving $1 \%$ triton $\mathrm{X}-100$ in erythrocyte solutions. The negative control test, in which erythrocytes are not lysed, was made by suspending erythrocytes in regular saline solution in previously sterile Eppendorf tubes. The samples were incubated for at least 15 minutes at $37^{\circ} \mathrm{C}$. Nearly $200 \mu \mathrm{L}$ samples were removed at specific pre-determined time intervals, i.e., 0.5, 1, 2, 4,5, $6,7,8,9$, and $10 \mathrm{hr}$, thus centrifuging at $1345 \times \mathrm{xg}$ for 15 minutes. A $100 \mu \mathrm{L}$ supernatant was incubated for 45 minutes at room temperature to achieve adequate haemoglobin to oxyhaemoglobin conversion. At $580 \mathrm{~nm}$, the absorbance was measured spectrophotometrically. The following formula is used to calculate the proportion of haemolysis:

$$
\% \text { Haemolysis }=\frac{A \text { sample }- \text { A spontaneous control }}{\text { A positive control }} \times 100
$$

Where, $\mathrm{A}_{\text {sample }}$ is the absorbance of the test sample containing the drug nanoparticles. A spontaneous control is the absorbance of erythrocytes that are previously incubated with saline water. On the other hand, A positive control was the absorbance of the supernatant of erythrocytes which was exposed with $1 \%$ Triton X-100 solution made up of normal saline. The experiment was performed in triplicate, and the data were expressed in mean $\pm \operatorname{SD}(n=3)$.

\subsection{Evaluation of erythrocyte membrane integrity:}

Lactate dehydrogenase ( $\mathrm{LDH})$ is an enzyme that is produced by erythrocytes and can be determined photometrically with the LDH assay kit / Lactate Dehydrogenase Assay Kit (Colorimetric) (ab102526)[42, 43]. The erythrocyte suspension was prepared according to the protocol previously discussed on haemolysis. $1 \mathrm{~mL}$ of erythrocyte suspension was used to administer the drug's optimised formulation, the drug, and the placebo. The positive control sample (100 percent lysed erythrocytes) was made by diluting the erythrocyte suspension with $1 \%$ Triton-X-100, while the negative control sample was made by diluting the erythrocyte suspension with normal saline solution. To allow LDH natural preparation, 150/UL Lactate dehydrogenase $(\mathrm{LDH})$ was incubated with erythrocyte suspension at $37^{\circ} \mathrm{C}$. During specific time 
periods, such as $2 \mathrm{~h}, 4 \mathrm{~h}$, and $8 \mathrm{~h}$ from suspension, $400 \mathrm{~L}$ samples were extracted and independently centrifuged at $1345 \mathrm{xg}$ for 20 minutes. $\mathrm{LDH}$ was detected at $500 \mathrm{~nm}$ after the supernatant was prepared with a ready-to-use LDH solution. The sum of LDH was calculated using the formula below.

$$
\text { Lactate dehydrogenase }(\mathrm{U} / \mathrm{L})=\frac{\text { Asample }- \text { A negative control }}{\text { A standard }} \times 150
$$

Where, $\mathrm{A}_{\text {sample stands }}$ for drug and nanoparticles incubated erythrocytes absorbance, $\mathrm{A}$ negative control indicates, erythrocytes absorbance of sample, which was pretreated with normal saline solution. The A standard indicates the absorbance of erythrocytes sustention, which were pretreated 150/UL Lactate dehydrogenase (LDH) enzyme. The experiment was performed in triplicate and the date is expressed as mean $\pm \mathrm{SD}(\mathrm{n}=3)$

\subsection{Platelet Aggregation Studies:}

Platelet aggregation experiments were performed using a haematological counter (Biolinx Lab systems Private Limited, India)[44]. Before platelet counting, $2 \mathrm{~mL}$ of blood was incubated with different drug concentrations for 3 hours at $37 \mathrm{oC}$, i.e. 10,50 , and $100 \mathrm{~g} / \mathrm{mL}$. After incubation, the required volume of sample was diluted with standard saline water, and additional samples were examined using a triplicate haematological counter (mean $\mathrm{SD} ; \mathrm{n}=3$ ). It is important to do a detailed study of platelet aggregation, which can be performed with an optical microscope. In this experiment, heparinized blood samples were treated with various samples. After treatment with the samples, peripheral blood smears were mounted on a glass slide and air-dried for 5 minutes. With the aid of Leishman's dye, the blood smears were pigmented for 10 minutes. The stain was then washed with purified water and covered with a glass cover. The staining essence of platelets was examined using an optical microscope, and high-resolution images were captured. 


\section{Results and discussion:}

\subsection{Factorial design for optimization:}

As seen in Table 1, a total of thirteen formulations (F1-F13) were created, and the optimization method was developed using the Box-Behnken design. The influence of different independent or input variables such as phosphatidylcholine concentration (X1), glyceryl tripalmitate (X2), and PLGA quantity (X3) on dependent or outcome variables such as particle size (Y1), zeta potential (Y2), and entrapment efficacy (Y3), brain drug concentration at 4h interval (Y4), and AUC(Brain)/AUC(Plasma)(Y5) was investigated. Fig. 2(A\&B) applies to the outcome of the results . In the F1 to F13 batches, the mean particle size of the prepared nanoparticles ranged from $173.51 \mathrm{~nm}(\mathrm{~F} 1)$ to $233.37 \mathrm{~nm}$ (F1) (F7). The range of possible Zeta values was discovered to be between $25.78 \mathrm{mV}$ (F11) and $36.31 \mathrm{mV}$. (F6). Drug entrapment efficacy (\%) ranged from 70.56 $\%$ (F8) to $86.34 \%$ (F9) (F10). The concentration of drugs in the brain was observed to be smaller in F5(6.38 g/mL) administration at the 4h time period, while it was higher in F6 $(12.38 \mathrm{~g} / \mathrm{mL})$ administration. Similarly, the AUC(Brain)/AUC(Plasma) ratio of the formulation varied from F5 (1.02) to F4 (4.34).

The relationship between a dependent variable (Y) and an independent variable (X) was studied statistically. The Design of Experts software was used to measure the analysis of variance (ANOVA), polynomial equation data, the fisher's value (F-value), the degree of freedom (df), the total of the squares, and the mean sum of the squares (Version 11.0).

For particle size $\left(\mathrm{Y}_{1}\right)$ measurement; the following polynomial equation was obtained:

$\operatorname{Particle} \operatorname{Size}(Y 1)=193.34-5.41 X_{1}+1.03 \mathrm{X}_{2}+20.49 X_{3}+1.72 X_{1} X_{2}-4.6475 X_{1} X_{3}+5.8375 X_{2} X_{3}-$

$$
6.64 X_{1}^{2}+5.15 X_{2}^{2}+8.166 X_{3}^{2}
$$

In Fig. 3[I], the influence of all independent variables on particle size (Y1) was addressed (a). The steep slope or curvature of factor 'C' or PLGA in Fig.3[I](a) suggests that concentrated PLGA will have a greater impact on particle size. Increased phosphatidylcholine concentration reduces particle size, as seen in the 3D surface plot of Fig. 3[I](b), indicating a negative coefficient of phosphatidylcholine in polynomial equation-1 (-5.41). Reduced PLGA concentration and 
increased Glyceryl tripalmitate concentration result in smaller particles, as seen in Fig.3[I] (c \& d).

For the zeta potential $\left(\mathrm{Y}_{2}\right)$ measurement; the following polynomial equation was obtained:

Zeta potential $\left(Y_{2}\right)=+32.56+4.02 X_{1}-0.5538 X_{3}+0.3600 X_{1} X_{2}+0.9325 X_{1} X_{3}+1.91 X_{2} X_{3}-1.00$

$$
\mathrm{X}_{1}^{2}-\mathbf{0 . 8 1 5 0} \mathrm{X}_{2}^{2}-\mathbf{0 . 4 2 7 5} \mathrm{X}_{2}^{2}
$$

Phosphatidylcholine (X2); factor "A" has a steep slope, meaning that phosphatidylcholine concentration has a greater effect on zeta potential, according to the perturbation plot (Fig.3[II](a) This may be attributed to the fact that phosphatidylcholine is a surfactant. It was verified by Fig.3[II] (b-c) and the polynomial equation-2 that raising the phosphatidylcholine concentration would increase the cationic nature of the formulation and increase the zeta potential. However, it was also observed from polynomial equation-2 that phosphatidylcholine has a noticeable impact on zeta potential as compared to PLGA and glyceryl tripalmitate alone. This may be attributed to the inclusion of amino groups in phosphatidylcholine as well as proper fabrication of the tripalmitate group on the surface of drug-loaded polymeric-lipid hybrid nanoparticles (PLHNPs). Where else, it can be estimated from Fig.3[II](d) that increasing the concentration of glyceryl tripalmitate would have a mild effect on zeta potential.

For Effects of Entrapment efficacy $\left(\mathrm{Y}_{3}\right)$; the following polynomial equation obtained:

Entrapment efficacy $\left(Y_{3}\right)=+76.26-2.13 X_{1}-1.11 X_{2}+5.26 X 3+0.7150 X_{1} X_{2}-1.77 X_{1} X_{3}-1.16$

$$
X_{2} X_{3}-1.70 X_{1}^{2}-1.36 X_{2}^{2}+2.51 X_{3}^{2}
$$

The perturbation plot (Fig.3[III](a) reveals that PLGA; factor "C" has a steep slope, suggesting that PLGA concentration has a greater effect on entrapment efficacy. As can be seen in Fig. 3[III](b), glyceryl tripalmitate has little effect on Entrapment efficacy. However, the polynomial equation-3 and Fig.3[III] (c-d) explicitly show that increasing the concentration of PLGA increased entrapment efficacy; this is attributed to the polymeric gelation of PLGA. For effects of Concentration of Drug in the brain at 4h interval (Y4); following polynomial equation obtained: 


\section{Concentration of drug in brain at $4 \mathrm{~h}$ interval $\left(\mathrm{Y}_{4}\right)=+8.24+1.16 \mathrm{X}_{1}+0.1837 \mathrm{X}_{2}+1.55 \mathrm{X}_{3^{-}}$}

$$
0.3075 X_{1} X_{2}-0.1400 X_{1} X_{3}-0.7800 X_{2} X_{3}+0.5738 X_{1}^{2}-0.2512 X_{2}^{2}+1.01 X_{3}^{2}
$$

The perturbation plot (Fig. 3[IV](a) shows that PLGA; factor "C" has a steep slope, meaning that PLGA concentration has a stronger effect on drug concentration in the brain at the $4 \mathrm{~h}$ interval. According to Fig.3[IV](b), glyceryl tripalmitate has no impact, whereas elevated phosphatidylcholine concentration has an agonistic effect on drug concentration in the brain at a 4h interval; this may be attributed to the role of phosphatidylcholine enhancing the active transport function of the drug in brain endothelial cells. However, it was clear from Fig.3[IV] (cd) and the polynomial equation-4 that an improvement in PLGA and phosphatidylcholine concentration has a significant impact on opioid concentration in the brain at the 4th hour interval. Effects of AUC (Brain)/AUC (Plasma)( $\left.\mathrm{Y}_{5}\right)$; following polynomial equation obtained:

Effects of AUC (Brain)/AUC (Plasma) $\left(Y_{5}\right)=+1.84+0.5638 X_{1-0.2350} X_{2}+1.12 X_{3-}$

$$
0.2525 X_{1} X_{2}+0.3000 X_{1} X_{3}-0.7225 X_{2} X_{3}+0.4025 X_{1}^{2}-0.3250 X_{2}^{2}+0.9425 X_{3}^{2}
$$

Once again, the perturbation plot (Fig.3[V](a) showed that factor " $\mathrm{C}$ " has a steep slope, suggesting that PLGA Concentration will have a stronger impact on the Effects of AUC (Brain)/AUC (Plasma) (Y5). According to Fig.3[V](b), glyceryl tripalmitate has no significant effect on AUC (Brain)/AUC (Plasma); however, an increase in phosphatidylcholine concentration could increase drug AUC in the brain; this could be due to the presence of the choline group, which helps drugs cross the blood-brain barrier by speeding up acetylcholine synthesis and boosting cognitive function of the brain. According to Fig. 3[V](c-d) and the polynomial equation-5, PLGA improves medication transport in the brain and has a better brain-protective impact.

As a result, increasing the PLGA concentration improved the AUC (Brain)/AUC (Plasma) ratio. Furthermore, the positive and negative signs of the interaction terms, as well as the co-efficient of the key outcomes, were used to denote the synergistic and antagonistic effects of the result variables in the polynomial equation 1-5. The estimation of the p-value and F-value (Table 2) often aids in determining the model's importance. The p-value was calculated at a 95\% confidence 
level (0.05). The $\mathrm{R}^{2}$ value and modified $\mathrm{R}^{2}$ value of a model must be close to 1 in order for it to be relevant. Furthermore, the lowest Bayesian Information Criterion (BIC) and Akaike Information Criterion (AIC) must be used; the AIC aids in determining the optimal consistency and goodness of fit standard. In comparison to another quadratic model of dependent variables, Table 2 shows the medication in the brain at $4 \mathrm{~h}$ interval (Y4) is the better fit model, with lower AIC (104.38) and BIC (0.0306) values and important R2 (0.9972) and modified R2 value (0.9887). Y1 (358.78) and Y4 (117.20) have higher F-values, meaning that experimental variation is much greater than error variance.

To bring more specification in the optimization process by Design of Expert (Version 11.0) software, the process capability index (Cpk) was identified in desirability studies (Fig. 4(a)). The constraint variables were obtained, i.e., particle size at $193.143 \mathrm{~nm}$., zeta potential at $33.7515 \mathrm{mV}$., entrapment efficiency $74.7937 \%$, Concentration of Drug in the brain at $4 \mathrm{~h}$ interval at

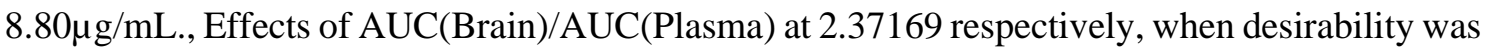
0.988.

From the overlay plot (Fig.4(b)) the desire region for constraining variables was identified within the yellow design space. Considering overlay plot's independent variables (phosphatidylcholine $(\mathrm{X} 1)=130.025 \&$ Glyceryl tripalmitate $(\mathrm{X} 2)=151.348)$ as a standard, three batches were reproduced. The three batches' dependent variables or outcome variable's relative standard error was found to be less than $9.00 \%$. Therefore, the formula obtained from the overly plot was considered as optimum.

\subsection{Characterization of PLHNPs:}

\subsubsection{In-vitro drug release study:}

Methotrexate-loaded polymeric-lipid hybrid nanoparticles (PLHNPs) exhibit unusual transport characteristics, including swelling and regulated release. For in-vitro drug release studies, the optimised batch from the overlay plot was used. Within 10 hours, the nanoparticles show initial burst effects and hance; $37.232 .35 \%$ of the drug was released. The existence of drug particles on the nanoparticle surface could explain initial drug release phenomenon. Phosphatidylcholine, in 
the presence of $0.75 \%$ tween 80 and a $\mathrm{pH} 7.4$ phosphate buffer solution, can facilitate Methotrexate release from the PLGA matrix by forming a canal. The zero-order kinetics was detected as the polymer degraded. After 72 hours or 3 days, nearly $49.373 .56 \%$ cumulative drug release was reported, suggesting a continuous release trend of the nanoparticles, where the correction coefficient $\left(\mathrm{R}^{2}\right)$ for the zero-order fit model was 0.9954 (Fig.5(a)).

\subsubsection{Fourier transform infrared spectroscopy:}

To identify the chemical bonds and functional group heterogeneity within the samples, Fourier transform infrared spectroscopy (FTIR) studies must be performed where the samples absorb selective absorption of infrared radiation. The FTIR studies help us to discover drug-polymer interactions. In Fig.5b, the pure compound, Methotrexate, PLGA, placebo, physical combination, and optimised PLHNPs all have a characteristic spectrum peak were mentioned. The N-H stretch of amide R-CO-NH2, carboxylic acid's $\mathrm{C}=\mathrm{C}-\mathrm{CO}-\mathrm{OH}$ stretch, and asymmetric aromatic $\mathrm{N}-\mathrm{O}$ stretching is all found in the Methotrexate peak at $3401.12 \mathrm{~cm}-1,2957.05 \mathrm{~cm}-1,1646.11 \mathrm{~cm}-1$, and $1525.18 \mathrm{~cm}-1$, respectively, indicating the existence of $\mathrm{N}-\mathrm{H}$ stretch of amide R-CO-NH2, carboxylic acid's C=C-CO-OH stretch, PLGA exhibits a typical peak at $2977.37 \mathrm{~cm}-1,1767.05$ $\mathrm{cm}-1,1404.24 \mathrm{~cm}-1$, and $1172.05 \mathrm{~cm}-1$, indicating the existence of the R-CO-OH group $\mathrm{C}=\mathrm{O}$ stretch of ester, C-O stretch, and carboxylic acid RCO-OH stretch, respectively. One point is evident from the IR spectrum of the placebo and physical mixture: there is no significant molecular activity that could change the chemical composition of the substances. The absence of any chemical reaction during fabrication is confirmed by the FTIR spectra of optimised PLHNPs. The N-H stretch at $3410.80 \mathrm{~cm}-1$ and the ester $\mathrm{C}=\mathrm{O}$ stretch at $1747.70 \mathrm{~cm}-1$, on the other hand, were observed to have lower intensity and transmittance; this was attributed to the drug's adequate fabrication with polymer and lipid. This property further establishes Methotrexate's salt existence within stable PLHNPs

\subsubsection{Thermal characteristics:}

Differential calorimetry scanning (DSC) was conducted to clarify the drug's crystalline and amorphous behaviour. Often allows to identify drug association with polymer. Fig.5(c) showed 
Methotrexate's DSC thermogramic peaks at $120.04^{\circ} \mathrm{C}$ and $238.31^{\circ} \mathrm{C}$. The placebo endothermic melting peak showed glyceryl tripalmitate melting point. PLGA's transition temperatures (Tg) rose in placebo due to the presence of glyceryl tripalmitate, and two endothermic peaks were found at $109.26^{\circ} \mathrm{C}$ and $248.65^{\circ} \mathrm{C}$. In optimised PLHNPs, the PLGA melting point peak was observed at $109.17^{\circ} \mathrm{C}$, but the peak intensity was poor compared to placebo, confirming optimised PLHNPs amorphous nature. Additionally, the physical condition of the substance was probably altered during encapsulation, so the formulation exhibits amorphous characteristics.

\subsubsection{X-ray diffraction study:}

From diffractogram Fig.5(d) the characteristic $2 \Theta$ value for methotrexate pure drug was observed in $16.96^{\circ} \mathrm{C}, 21.22^{\circ} \mathrm{C}, 28.04^{\circ} \mathrm{C}$. From extremely imminent peaks and some tiny peaks, methotrexate may be expected to exhibit crystalline form. The diffractogram of placebo reveals the signature $2 \Theta$ value at $11.52^{\circ} \mathrm{C}, 19.41^{\circ} \mathrm{C}, 21.21^{\circ} \mathrm{C}, 27.66^{\circ} \mathrm{C}$, and $31.13^{\circ} \mathrm{C}$. Placebo diffractogram also showing low-intensity partial crystalline nature, due to the inclusion of PLGA, phosphatidylcholine \& glyceryl tripalmitate in placebo sample. The configured diffractogram of PLHNPs reveals characteristic $2 \Theta$ at $10.0^{\circ} \mathrm{C}, 19.66^{\circ} \mathrm{C} \& 27.26^{\circ} \mathrm{C}$. The reduced amplitude of optimised polymeric-lipid hybrid nanoparticles (PLHNPs) diffractogram peaks reveals the amorphous existence and proper encapsulation of drugs within optimised PLHNPs.

\subsubsection{Transmission electron microscopy:}

With the help of JEM-ARM300F GRAND ARM Atomic Resolution Electron Microscope, it was possible to focus on drug-loaded PLHNPs, which were placed on cooper coated gride (300 mesh). Fig.5(e) revealed that the projected particle was uniform in size and spherical in nature. The projected nanoparticle in TEM analysis was found to have lesser particle size as compared to particles size, which was obtained from the Delsa Nano C instrument (Beckman Coulter, USA); this is because Delsa Nano C identifies the apparent volume of distribution of the diluted particles in distilled water, however during TEM measurement, the particle may get dehydrated. Moreover, the chances of aggregated particles while measuring in Delsa Nano C instruments was formidable, as prior measurement, the nanosuspension was exposed to 15 minutes sonication. 


\subsubsection{Determination of brain uptake potential:}

A particular HPLC method was developed from the brain homogenates and plasma samples of experimental animals, i.e. Wister rates, which appeared to have a high degree of specificity and precision at a specific time interval. After 4 hours of drug administration, the $A U C_{\text {brain }} / \mathrm{AUC}_{\text {Plasma }}$ drug concentration in plasma was measured. The drug concentration in the brain was estimated to be $2.43 \mu \mathrm{g} / \mathrm{g}$ after 4 hours of administration, and the $\mathrm{AUC}_{\text {brain }} / \mathrm{AUC}_{\text {Plasma }}$ of the drug was found to be 2.23 after 4 hours of administration. This implies the drug concentration in the brain was 2.23 times greater than the drug concentration in plasma, which was absorbed by intravenous route. As a result, Methotrexate may be targeted in the brain in a passive manner.

\subsubsection{Cytotoxicity studies against U-87 MG glioma cells:}

To compare the efficacy of Methotrexate against U-87 MG glioma cell lines, cytotoxicity tests were carried out. Methotrexate, placebo-PLHNPs, and optimized-PLHNPs cytotoxicity against 87 MG glioma cell lines can be seen in Fig.6 (a). In all concentrations, the cellular cytotoxicity of Methotrexate loaded Optimized-PLHNPs was found to be significantly higher than that of free Methotrexate (p0.05). The cytotoxicity study's most unexpected result was that placebo- PLHNPs exhibit cytotoxicity when concentration rises. According to Sima Rezvantalab et al., (2018) this may be attributed to the existence of PLGA in the formulation. According to the article, PLGAbased nanoparticles have promising anticancer properties..

\subsubsection{Cellular uptake results:}

The U-87 MG glioma cells were picked up with optimized- PLHNPs, and stained with DAPI reagent, as shown in Fig.6(b). Suitable filters were used to capture the CLSM representation of DAPI reagent. After 4 hours of incubation with U-87 MG glioma cells, the image indicates extensive internalisation of optimized- PLHNPs, It's possible that nanoparticles can clump together in the cytoplasm. Many apoptotic processes, such as the expression of caspase-3 mRNA, the downregulation of metalloproteinases (MMPs), the upregulation of $\mathrm{p} 53$, and the deactivation of IAP5, are believed to take place in the cytoplasm, according to various reports. As a consequence, optimized- PLHNPs, may be an useful tool to induce cytotoxicity in the cytoplasm. 


\subsubsection{Evaluation of haemolysis:}

The most important study to learn about the behaviour of prepared PLHNPs with blood was an in-vivo toxicity study. The association of PLHNPs with blood and its substances must be investigated. The PLHNPs of PLGA, which are made up of lactide (LA) and glycolide (GA) monomers, have a minor impact on homolyses. The findings of the analysis suggest that PLHNPs can be used with care. The profile of percentage hemolysis absorbance with 0.5 th and 10th-hour shifts of erythrocytes with different treatments $(10,50,100 \mathrm{~g} / \mathrm{ml})$ containing Methotrexate, placebo-PLHNPs, and Methotrexate primed optimised PLHNPs is depicted in Fig.7(a-d). It can be inferred from Fig.7(d) that placebo-PLHNPs and Methotrexate-loaded optimised PLHNPs cause fewer haemolysis than Methotrexate. Regardless of Drug and PLHNPs concentrations of $10,50,100 \mathrm{~g} / \mathrm{mL}$, the placebo- PLHNPs and Methotrexate-loaded optimised PLHNPs displayed fewer hemocompatibility (>1\%) and were found to be safe to RBC membrane integrity. Most notably, Methotrexate-loaded optimised PLHNPs formulations display fewer haemolysis; this is attributed to adequate Methotrexate encapsulation inside the PLHNPs, which prevents red blood cells from Methotrexate-induced haemolysis. One thing that can be proven from the haemolysis studies is that i.v. administration of Methotrexate primed optimised PLHNPs can have a beneficial impact on erythrocytes. As a consequence, there's a decent risk of lowering malondialdehyde (MDA) levels and carbonyl group content. As a consequence, methotrexate-loaded optimised PLHNPs can prevent erythrocyte degradation during glioma care when delivered intravenously.

\subsubsection{Evaluation of erythrocyte membrane integrity:}

White blood cells and blood plasma were separated using centrifugation, and natural saline comprising erythrocytes was used instead. Enumerating the LDH enzyme is expected to determine membrane integrity. Only when the structural integrity of the erythrocytes was disrupted was a hyper or elevated degree of LDH recorded. Figure 8(a-c) shows the sum of LDH released after treatment with $2 \mathrm{~mL}$ of erythrocyte suspension with Methotrexate, methotrexateloaded optimised PLHNPs (equivalent to 10,50 , and $100 \mathrm{~g} / \mathrm{mL}$ of Methotrexate), and placeboPLHNPs. By matching placebo-loaded optimised PLHNPs of 10, 50, and $100 \mathrm{~g} / \mathrm{mL}$ concentration 
at 8th-hour intervals to Methotrexate-loaded methotrexate-loaded optimised PLHNPs of 10, 50, and $100 \mathrm{~g} / \mathrm{mL}$ concentration, the LDH release was not as conspicuous (20\%). Triton X 100, on the other side, displays approximately $290 \%$ LDH release, suggesting total erythrocyte annihilation. As a result, methotrexate-loaded optimised PLHNPs are unlikely to damage erythrocyte membrane integrity.Thus, methotrexate-loaded optimised PLHNPs will be suitable for intravenous administration.

\subsubsection{Platelet aggregation study results:}

Patients can experience myocardial infarction, ischemia, or stroke after receiving nanoparticles from i.v. routs; these problems can occur as a result of platelet aggregation that leads to thrombus formation. As a result, platelet aggregation must be assessed after i.v. administration of Methotrexate, Placebo-PLHNPs, and Methotrexate-loaded optimised PLHNPs. The quantitative estimation of platelets was measured by hematological counter after incubating with Methotrexate, Placebo- PLHNPs and Methotrexate loaded optimized PLHNPs at 10, 50, 100 $\mu \mathrm{g} / \mathrm{mL}$ concentration for $3 \mathrm{hr}$ at $37^{\circ} \mathrm{C}$. The $\mathrm{pH} 6.4$ phosphate buffer was considered a stand-alone control group throughout the study. At all Concentration, the Methotrexate did not show any significant differences in platelet count as compared to the control pH 6.4 phosphate buffer (PBS) solution, which postulating Methotrexate did not show any platelet aggregation (Fig.9(a)). The Placebo- PLHNPs showed significantly lower $(\mathrm{p}<0.005)$ platelet count than that of Methotrexate \& Methotrexate loaded optimized PLHNPs treated samples. However, $100 \mu \mathrm{g} / \mathrm{mL}$ of Methotrexate loaded optimized PLHNPs shows higher platelet aggregation compare to PlaceboPLHNPs, which indicates a non-significance $(\mathrm{p}>0.05)$ relationship. Lower platelet aggregation for Placebo- PLHNPs was due to the absence of Methotrexate, which may influence platelet aggregation. The light microscopy images of Leishman's-stained whole blood samples after treating with different Concentration of pH 6.4 phosphate buffer solution, Methotrexate, PlaceboPLHNPs, and Methotrexate loaded optimized PLHNPs was shown in Fig.9(b). 


\section{Discussion:}

The present study demonstrated the in-vitro investigation and systematic optimization of Methotrexate loaded PLHNPs using Box-Behnken design. The optimization process seeks to provide adequate particle size, zeta potential, entrapment efficacy with maximum drug ratio in the brain. From the optimization process, it was also understood that the increased concentration of PLGA and glycerol tripalmitate helps to increase the particle size.On the other hand, increase the Concentration of phosphatidylcholine could increase the zeta potential(cationic) of the formulation, which is due to the presence of the L-alpha-phosphatidylcholine group. Noticeably, increase concentration of PLGA improvises drug entrapment efficacy (\%), and increase concentration of PLGA and phosphatidylcholine increases drug concentration in the brain at $4^{\text {th }}$ interval; as per Sandeep Godaraa et al. (2019) while preparing paclitaxel PLGA PLHNPs, it was observed that coating of lipid over nanoparticles could enhances paclitaxel blood circulation time as compared to uncoated nanoparticles. Which is due to the restriction of plasma protein adsorption over lipid corona coating[45].

As per recent research done by Vanesa Noz et al. (2021) PLGA nanoparticles improve dopamine transmission to parkinson's disease rats and shield dopamine from fast cortical metabolism[46]. In this current research, increase Concentration of PLGA could improve the effects of the AUC(Brain)/AUC(Plasma) ratio. Therefore, optimization of PLGA concentration was essential. Upon optimization, the resultant formulation shows excellent sustain release property in in-vitro drug release studies for $72 \mathrm{hr}$, which would be beneficial for prolonged action. From the FTIR, DSC and XRD analysis, it was confirmed that no such potential interaction took place between Methotrexate and other components, and the Methotrexate loaded optimized PLHNPs show amorphous nature.

From the observation of transmission electron microscopy, it was concluded that Methotrexate loaded optimised PLHNPs showed good spherical shape with the absence of mushroom and ostwald ripening effect, indicating higher stability as formulation. 
From the cytotoxicity studies, it was confirmed that Methotrexate loaded optimized PLHNPs produces significantly higher cytotoxicity in U-87 MG glioma cell lines than that of free Methotrexate $(\mathrm{p}<0.05)$; This may be attributed to the combined effects of Methotrexate and PLGA, but more importantly due to the improved stable structure of PLHNPs after absorption of PVA, as the drug diffusion through the cellular matrix is well regulated and stable, and therefore indirectly this phenomenon could improve the anticancer activity of PLHNPs. As per Iliyas Khan et al. (2016) Polylactide-co-glycolide (PLGA) is a biodegradable polymer used widely to produce NPs for drug delivery[47].Extensive application of PLGA NPs promises cancer treatment, with better efficacy and with fewer side effects.

A confocal laser scanning microscopy confirmed that Methotrexate-loaded optimized PLHNPs show excellent internalization in U-87 MG glioma cell lines, which was approved by observing highly concentrated cytoplasm in 4',6-diamidino-2-phenylindole (DAPI) reagent. Since the cytoplasm was an established site of action for any chemotherapeutic medicaments, hance, free Methotrexate rising from Methotrexate loaded optimized PLHNPs would exert higher anticancer efficiency. The brain uptake studies confirmed that passive brain targeting is possible with the Methotrexate-loaded optimized PLHNPs. From the haemolysis studies, it was witnessed that, Methotrexate loaded optimized PLHNPs exerts less than 1\% haemolysis; as per Justin M. Zook et al. (2011) the percentage of hemolysis decreases when prepared stable AgNP agglomerate size increases[48]. Since, in existing research the optimized PLHNPs shows much less than 200nm particles size, hance chances of hemolysis would be negligible. From the platelet aggregation studies, it was confirmed that platelets were less aggregated in the presence of Methotrexate loaded optimized PLHNPs. The optimized PLHNPs established itself as the most promising candidate for cancer teragating, therefore optimized PLHNPs would be a reliable drug delivery system for glioblastoma treatment. However, in vivo anticancer potential of methotrexate loaded optimized PLHNPs will need to be evaluated in future studies. 


\section{Conclusion:}

Methotrexate-loaded PLHNPs is synthesised using one-step solvent injection method. With higher drug content in the brain, PLHNPs with a lipid shell and a polymeric core display less hemolysis and platelet aggregation. It can be concluded that phosphatidylcholine and glyceryl tripalmitate coated with PLGA core matrix can open up a new way to deliver lipophilic constituents with increased potential for glioma treatment.

\section{Acknowledgment:}

I would like to acknowledge Dr. R.S. Gaud, Director, SVKM's NMIMS Deemed-to-be University, Shirpur Campus, for providing me with excellent research facilities and deep encouragement while pursuing this project. I would also like to acknowledge Bharathidasan University Tiruchirappalli Tamil Nadu-620024, India, for studying Confocal Laser Scanning Microscope (CLSM). I express my sincere gratitude to CSIR North East Institute of Science and Technology (NEIST) Jorhat-785006 Assam, India, for supporting me in my studies on Atomic Force Microscope (AFM).

\section{References:}

[1] Shi J, Votruba AR, Farokhzad OC, Langer RJNI. Nanotechnology in drug delivery and tissue engineering: from discovery to applications. 2010;10:3223-30.

[2] Li W, Szoka FCJPr. Lipid-based nanoparticles for nucleic acid delivery. 2007;24:438-49.

[3] Batrakova EV, Kabanov AVJJocr. Pluronic block copolymers: evolution of drug delivery concept from inert nanocarriers to biological response modifiers. 2008;130:98-106.

[4] Jasim A, Abdelghany S, Greish K. Current update on the role of enhanced permeability and retention effect in cancer nanomedicine. Nanotechnology-Based Approaches for Targeting and Delivery of Drugs and Genes: Elsevier; 2017, p. 62-109.

[5] Sur S, Rathore A, Dave V, Reddy KR, Chouhan RS, Sadhu VJN-S, Nano-Objects. Recent developments in functionalized polymer nanoparticles for efficient drug delivery system. 2019;20:100397.

[6] Jesus S, Schmutz M, Som C, Borchard G, Wick P, Borges OJFib, biotechnology. Hazard assessment of polymeric nanobiomaterials for drug delivery: what can we learn from literature so far. 2019;7:261.

[7] Rangsimawong W, Opanasopit P, Rojanarata T, Duangjit S, Ngawhirunpat TJB, Bulletin P. Skin transport of hydrophilic compound-loaded PEGylated lipid nanocarriers: comparative study of liposomes, niosomes, and solid lipid nanoparticles. 2016;39:1254-62. 
[8] Puri A, Loomis K, Smith B, Lee J-H, Yavlovich A, Heldman E, Blumenthal RJCRiTDCS. Lipidbased nanoparticles as pharmaceutical drug carriers: from concepts to clinic. 2009;26. [9] Mandal B, Bhattacharjee H, Mittal N, Sah H, Balabathula P, Thoma LA, Wood GCJNN, Biology, Medicine. Core-shell-type lipid-polymer hybrid nanoparticles as a drug delivery platform. 2013;9:474-91.

[10] Zhang L, Chan JM, Gu FX, Rhee J-W, Wang AZ, Radovic-Moreno AF, Alexis F, Langer R, Farokhzad OCJAn. Self-assembled lipid- polymer hybrid nanoparticles: a robust drug delivery platform. 2008;2:1696-702.

[11] Bertoni S, Passerini N, Albertini B. Nanomaterials for oral drug administration. Nanotechnology for Oral Drug Delivery: Elsevier; 2020, p. 27-76.

[12] Shi J, Xiao Z, Votruba AR, Vilos C, Farokhzad OCJACIE. Differentially charged hollow core/shell lipid-polymer-lipid hybrid nanoparticles for small interfering RNA delivery. 2011;50:7027-31.

[13] Gu J, Chen Y, Tong L, Wang X, Yu D, Wu HJJon. Astaxanthin-loaded polymer-lipid hybrid nanoparticles (ATX-LPN): assessment of potential otoprotective effects. 2020;18:1-17.

[14] Massadeh S, Omer ME, Alterawi A, Ali R, Alanazi FH, Almutairi F, Almotairi W, Alobaidi FF, Alhelal K, Almutairi MSJP. Optimized Polyethylene Glycolylated Polymer-Lipid Hybrid Nanoparticles as a Potential Breast Cancer Treatment. 2020;12:666.

[15] Ai X, Duan Y, Zhang Q, Sun D, Fang RH, Liu-Bryan R, Gao W, Zhang LJ, medicine t. Cartilage-targeting ultrasmall lipid-polymer hybrid nanoparticles for the prevention of cartilage degradation. 2021;6:e10187.

[16] Tahir N, Madni A, Balasubramanian V, Rehman M, Correia A, Kashif PM, Mäkilä E, Salonen J, Santos HAJljop. Development and optimization of methotrexate-loaded lipid-polymer hybrid nanoparticles for controlled drug delivery applications. 2017;533:156-68.

[17] Gardouh AR, Attia MA, Enan ET, Elbahaie AM, Fouad RA, El-Shafey M, Youssef AM, Alomar SY, Ali ZA-E, Zaitone SAJM. Synthesis and Antitumor Activity of Doxycycline Polymeric

Nanoparticles: Effect on Tumor Apoptosis in Solid Ehrlich Carcinoma. 2020;25:3230.

[18] Panday R, Poudel AJ, Li X, Adhikari M, Ullah MW, Yang GJC, Biointerfaces SB. Amphiphilic core-shell nanoparticles: Synthesis, biophysical properties, and applications. 2018;172:68-81.

[19] Sah AK, Suresh PK, Verma VKJAc, nanomedicine,, biotechnology. PLGA nanoparticles for ocular delivery of loteprednol etabonate: a corneal penetration study. 2017;45:1156-64.

[20] Luo Y, Yang H, Zhou Y-F, Hu BJJoCR. Dual and multi-targeted nanoparticles for site-specific brain drug delivery. 2020;317:195-215.

[21] Tapeinos C, Battaglini M, Ciofani GJJoCR. Advances in the design of solid lipid nanoparticles and nanostructured lipid carriers for targeting brain diseases. 2017;264:306-32. [22] Haggag YA, Ibrahim RR, Hafiz AAJljon. Design, formulation and in vivo evaluation of novel honokiol-loaded PEGylated PLGA nanocapsules for treatment of breast cancer. 2020;15:1625. [23] Yang V, Gouveia MJ, Santos J, Koksch B, Amorim I, Gärtner F, Vale NJRMC. Breast cancer: insights in disease and influence of drug methotrexate. 2020;11:646-64.

[24] Jang J-H, Jeong S-H, Lee Y-BJljoms. Preparation and in vitro/in vivo characterization of polymeric nanoparticles containing methotrexate to improve lymphatic delivery. 2019;20:3312.

[25] Bhaskarabhatla A. Is the 2013 Price Control Regulation Necessary? Regulating Pharmaceutical Prices in India: Springer; 2018, p. 33-67.

[26] Kim K-T, Lee J-Y, Kim D-D, Yoon I-S, Cho H-JJP. Recent progress in the development of poly (lactic-co-glycolic acid)-based nanostructures for cancer imaging and therapy. 2019;11:280.

[27] Dave V, Tak K, Sohgaura A, Gupta A, Sadhu V, Reddy KRJJomm. Lipid-polymer hybrid nanoparticles: Synthesis strategies and biomedical applications. 2019;160:130-42.

[28] Shaikh MV, Kala M, Nivsarkar MJEJoPS. Formulation and optimization of doxorubicin loaded polymeric nanoparticles using Box-Behnken design: ex-vivo stability and in-vitro activity. 2017;100:262-72. 
[29] Phalguna Y, Pothula S, Sarepalli RK, Murala B. Design, characterization and In vitro evaluation of polymeric nanoparticles containing decitabine. 2020.

[30] Surve DH, Jindal ABJJop, analysis b. Development and validation of reverse-phase highperformance liquid chromatographic (RP-HPLC) method for quantification of Efavirenz in Efavirenz-Enfuvirtide co-loaded polymer-lipid hybrid nanoparticles. 2019;175:112765.

[31] Behbahani ES, Ghaedi M, Abbaspour M, Rostamizadeh KJUs. Optimization and characterization of ultrasound assisted preparation of curcumin-loaded solid lipid nanoparticles: Application of central composite design, thermal analysis and X-ray diffraction techniques. 2017;38:271-80.

[32] Prajatelistia E, Sanandiya ND, Nurrochman A, Marseli F, Choy S, Hwang DSJCP. Biomimetic Janus chitin nanofiber membrane for potential guided bone regeneration application.

2021;251:117032.

[33] de Almeida Moura C, da Silva Lima JP, Silveira VAM, Miguel MAL, Luchiari ACJBp. Time place learning and activity profile under constant light and constant dark in zebrafish (Danio rerio). 2017;138:49-57.

[34] Bhattacharya SJljobm. Fabrication and characterization of chitosan-based polymeric nanoparticles of Imatinib for colorectal cancer targeting application. 2020;151:104-15.

[35] Bhattacharya SJAJoPFftafAJP. Design and Development of Docetaxel Solid SelfMicroemulsifying Drug Delivery System Using Principal Component Analysis and D-Optimal Design. 2018;12.

[36] Tan Y, Li XC, Liu XYJJotCCS. Chemoselective hydrogenation of 3-nitrostyrene over supported gold catalysts: Effect of loadings of gold. 2020.

[37] Tamura K, Kubo T, Nagaoki I, Mise H, Wakui A, Wayama M, Matsumoto H, Yaguchi TJM, Microanalysis. A 120 kV Transmission Electron Microscope Series for Both Life Science and Material Science Fields. 2018;24:1156-7.

[38] Hady MA, Sayed OM, AkI MAJC, Biointerfaces SB. Brain uptake and accumulation of new levofloxacin-doxycycline combination through the use of solid lipid nanoparticles: Formulation; Optimization and in-vivo evaluation. 2020;193:111076.

[39] Caetano-Pinto P, Jamalpoor A, Ham J, Goumenou A, Mommersteeg M, Pijnenburg D, Ruijtenbeek R, Sanchez-Romero N, van Zelst B, Heil SGJMp. Cetuximab prevents methotrexateinduced cytotoxicity in vitro through epidermal growth factor dependent regulation of renal drug transporters. 2017;14:2147-57.

[40] Moll G, Ankrum JA, Kamhieh-Milz J, Bieback K, Ringdén O, Volk H-D, Geissler S, Reinke PJTimm. Intravascular mesenchymal stromal/stem cell therapy product diversification: time for new clinical guidelines. 2019;25:149-63.

[41] Madani F, Esnaashari SS, Bergonzi MC, Webster TJ, Younes HM, Khosravani M, Adabi MJLS. Paclitaxel/methotrexate co-loaded PLGA nanoparticles in glioblastoma treatment: Formulation development and in vitro antitumor activity evaluation. 2020;256:117943. [42] Su J, Liu G, Lian Y, Kamal Z, Que X, Qiu Y, Qiu MJRa. Preparation and characterization of erythrocyte membrane cloaked PLGA/arsenic trioxide nanoparticles and evaluation of their in vitro anti-tumor effect. 2018;8:20068-76.

[43] Gurel A, Armutçu F, Damatoglu S, Unalacak M, Demircan NJEJGM. Evaluation of erythrocyte $\mathrm{Na}+, \mathrm{K}+-$ ATPase and superoxide dismutase activities and malondialdehyde level alteration in coal miners. 2004;1:22-8.

[44] Lee B-J, Jo I-Y, Bu Y, Park J-W, Maeng S, Kang H, Jang W, Hwang D-S, Lee W, Min KJJoe. Antiplatelet effects of Spatholobus suberectus via inhibition of the glycoprotein IIb/IIla receptor. 2011;134:460-7.

[45] Godara S, Lather V, Kirthanashri S, Awasthi R, Pandita DJMS, C E. Lipid-PLGA hybrid nanoparticles of paclitaxel: preparation, characterization, in vitro and in vivo evaluation. 2020;109:110576. 
[46] Nozal V, Rojas-Prats E, Maestro I, Gil C, Perez DI, Martinez AJIJoMS. Improved Controlled Release and Brain Penetration of the Small Molecule S14 Using PLGA Nanoparticles.

2021;22:3206.

[47] Khan I, Gothwal A, Sharma AK, Kesharwani P, Gupta L, Iyer AK, Gupta UJCRiTDCS. PLGA nanoparticles and their versatile role in anticancer drug delivery. 2016;33.

[48] Zook JM, MacCuspie RI, Locascio LE, Halter MD, Elliott JTJN. Stable nanoparticle aggregates/agglomerates of different sizes and the effect of their size on hemolytic cytotoxicity. 2011;5:517-30. 
Table 1: Box-Behnken design layout for the optimization of Methotrexate loaded PLGA lipid hybrid nanoparticles

\begin{tabular}{|c|c|c|c|c|c|c|c|c|}
\hline Formulation & $\begin{array}{c}\text { Phosphatidylcholine } \\
\text { (X1) }\end{array}$ & $\begin{array}{l}\text { Glyceryl } \\
\text { tripalmitate } \\
\text { (X2) }\end{array}$ & $\begin{array}{l}\text { PLGA } \\
\text { (X3) }\end{array}$ & $\begin{array}{l}\text { Particle } \\
\text { Size } \\
\text { (Y1) }\end{array}$ & $\begin{array}{c}\text { Zeta } \\
\text { Potential } \\
\text { (Y2) }\end{array}$ & $\begin{array}{c}\text { Entrapment } \\
\text { efficacy } \\
\text { (Y3) }\end{array}$ & $\begin{array}{l}\text { Concentration } \\
\text { of drug in } \\
\text { brain at } 4 \mathrm{~h} \\
\text { time interval } \\
\text { (Y4) }\end{array}$ & $\begin{array}{l}\text { AUC(Brain)/AUC(Plasma) } \\
\text { (Y5) }\end{array}$ \\
\hline & mg & mg & mg & $\mathbf{n m}$ & $\mathbf{m V}$ & $\%$ & $\mu \mathrm{g} / \mathrm{mL}$ & \\
\hline F1 & 150 & 175 & 200 & 173.51 & 34.42 & 71.35 & 9.54 & 2.12 \\
\hline F2 & 112.5 & 175 & 225 & 193.34 & 32.56 & 76.26 & 8.24 & 1.84 \\
\hline F3 & 75 & 175 & 200 & 175.32 & 27.81 & 71.41 & 6.99 & 1.65 \\
\hline F4 & 112.5 & 150 & 250 & 221.02 & 29.48 & 84.73 & 11.03 & 4.34 \\
\hline F5 & 112.5 & 150 & 200 & 191.62 & 33.78 & 72.73 & 6.38 & 1.02 \\
\hline F6 & 150 & 175 & 250 & 205.11 & 36.31 & 79.18 & 12.38 & 5.32 \\
\hline F7 & 112.5 & 200 & 250 & 233.37 & 32.67 & 79.76 & 10.06 & 2.45 \\
\hline F8 & 150 & 200 & 225 & 190.03 & 34.11 & 70.56 & 9.52 & 2.01 \\
\hline F9 & 112.5 & 200 & 200 & 180.62 & 29.34 & 72.42 & 8.53 & 2.02 \\
\hline F10 & 75 & 175 & 250 & 225.51 & 25.97 & 86.34 & 10.39 & 3.65 \\
\hline F11 & 75 & 200 & 225 & 197.12 & 25.78 & 74.03 & 7.75 & 1.33 \\
\hline F12 & 150 & 150 & 225 & 183.12 & 34.98 & 70.92 & 9.99 & 3.01 \\
\hline F13 & 75 & 150 & 225 & 197.12 & 28.09 & 77.25 & 6.99 & 1.32 \\
\hline
\end{tabular}


Table 2: ANOVA responses of prepared PLGA lipid hybrid nanoparticles

\begin{tabular}{|c|c|c|c|c|c|c|}
\hline \multicolumn{7}{|c|}{ Table 2: ANOVA responses of prepared PLGA lipid hybrid nanoparticles } \\
\hline Response variables & F-value & p-value & R2 & Adjusted R2 & BIC & AICc \\
\hline Particle size (Y1) & 358.78 & 0.0002 & 0.9991 & 0.9963 & 47.34 & 151.69 \\
\hline Zeta potential (Y2) & 52.67 & 0.0038 & 0.9937 & 0.9748 & 28.80 & 133.15 \\
\hline Entrapment efficacy (Y3) & 41.65 & 0.0054 & 0.9921 & 0.9682 & 41.89 & 146.24 \\
\hline Concentration of drug in brain at $4 \mathrm{~h}$ interval & 117.20 & 0.0012 & 0.9972 & 0.9887 & 0.0306 & 104.38 \\
\hline (Y4) & & & & & & \\
\hline Effects of AUC(Brain)/AUC(Plasma) (Y5) & 23.85 & 0.0122 & 0.9862 & 0.9449 & 12.21 & 116.56 \\
\hline
\end{tabular}




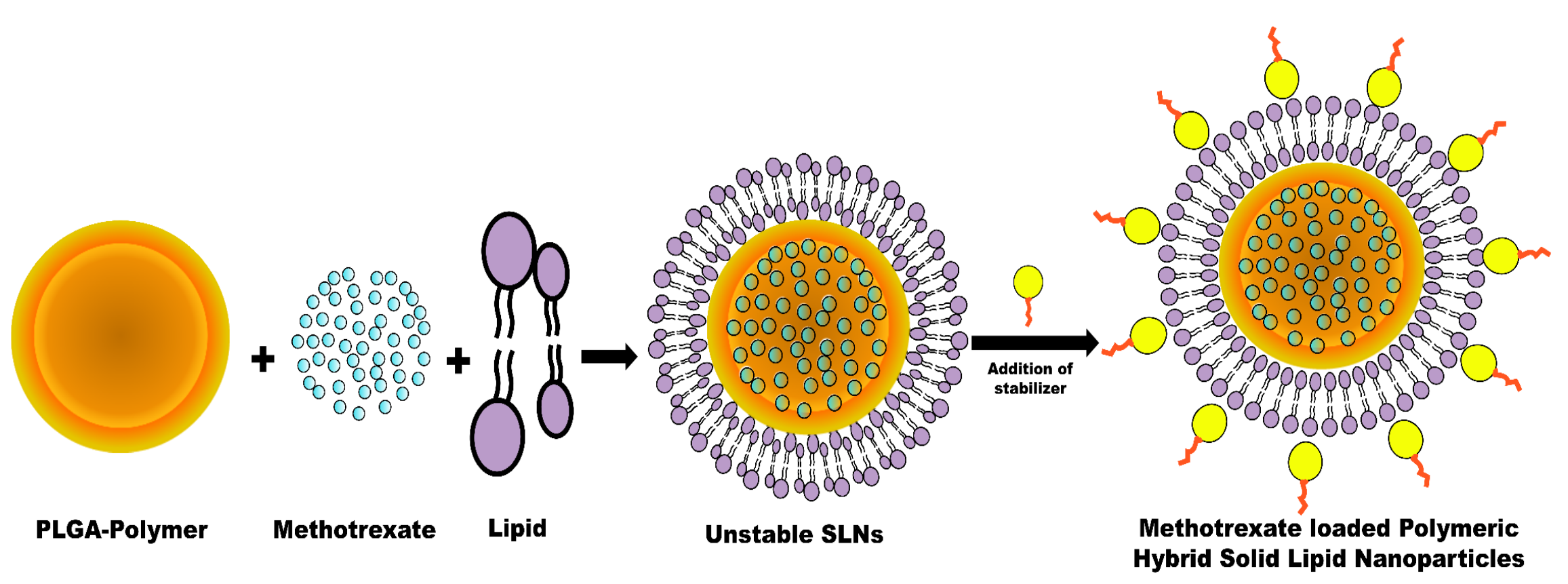

Fig.1: Schematic presentation of Methotrexate loaded poly (lactic-co-glycolic acid) lipid hybrid nanoparticle preparation and drug loading 


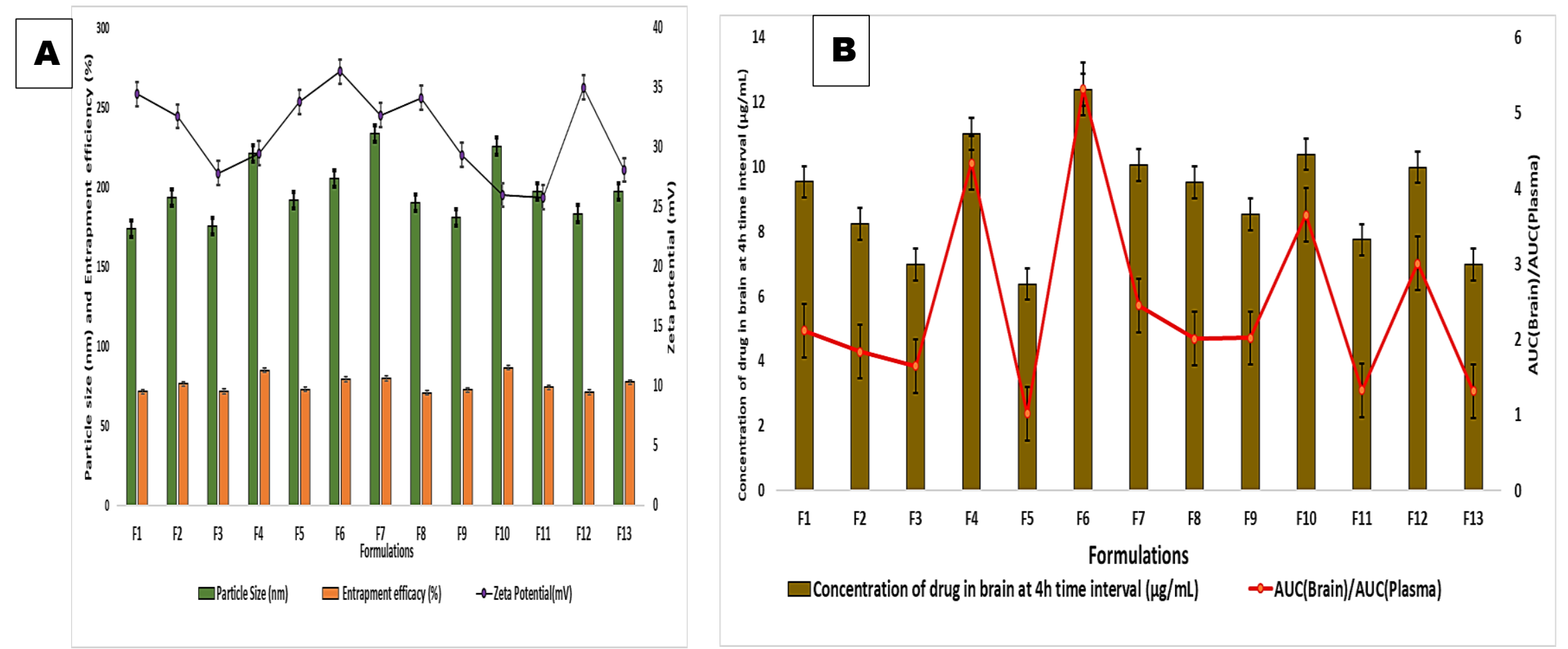

Fig.2: (A)Particle size, zeta potential and entrapment efficacy of formulations (F1 to F13) (B)Concentration in brain and brain/plasma ratio of drug and AUC(Brain)/AUC (Plasma) at $4 \mathrm{~h}$ interval 
a

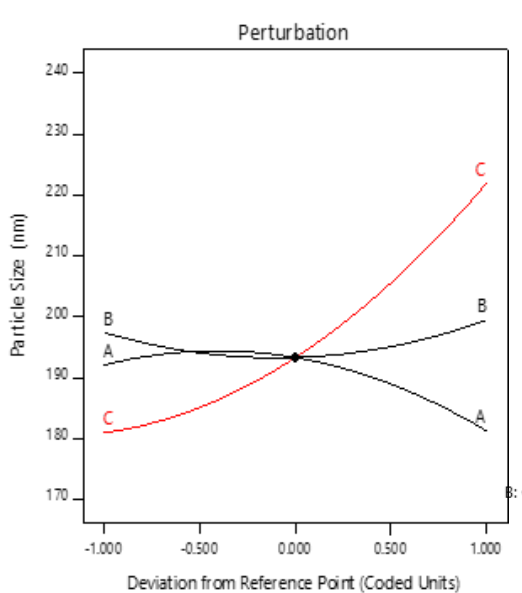

a

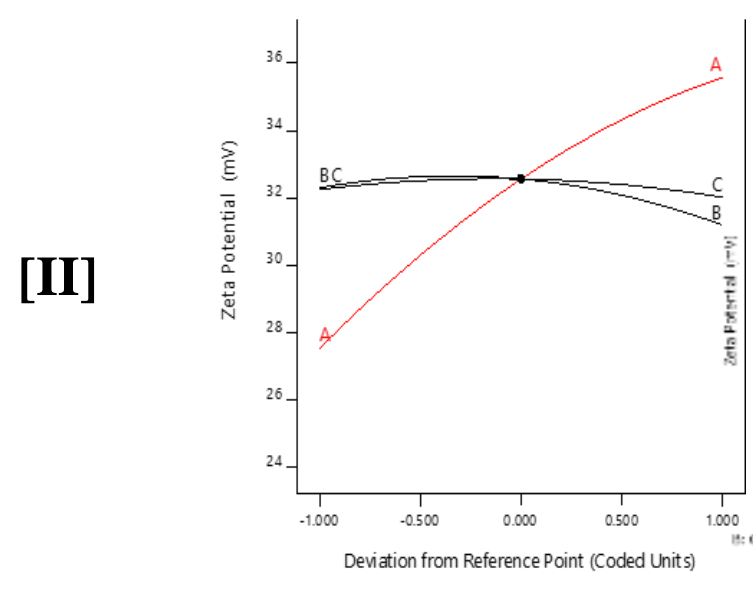

b

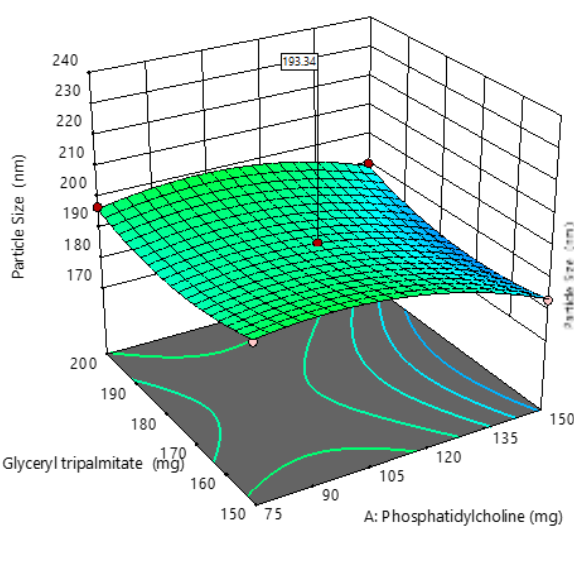

b

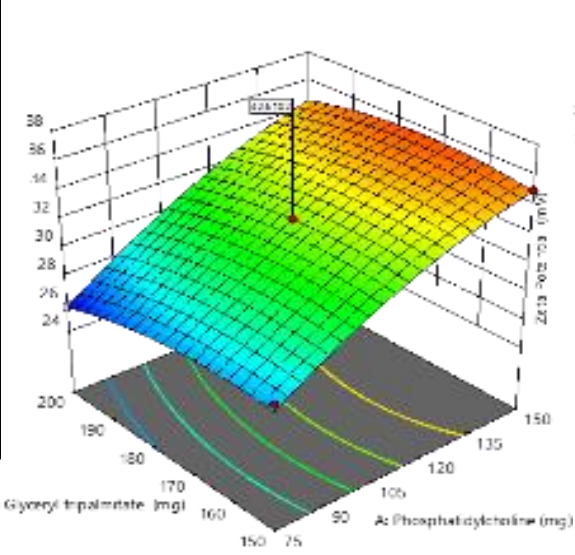

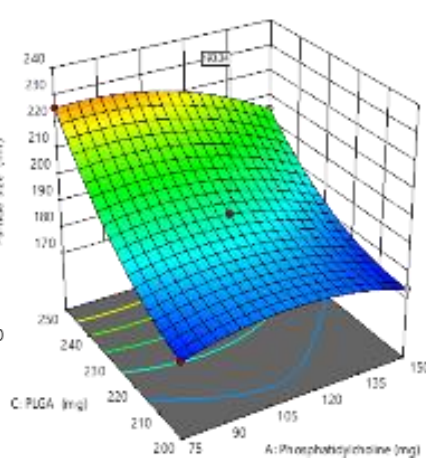

C
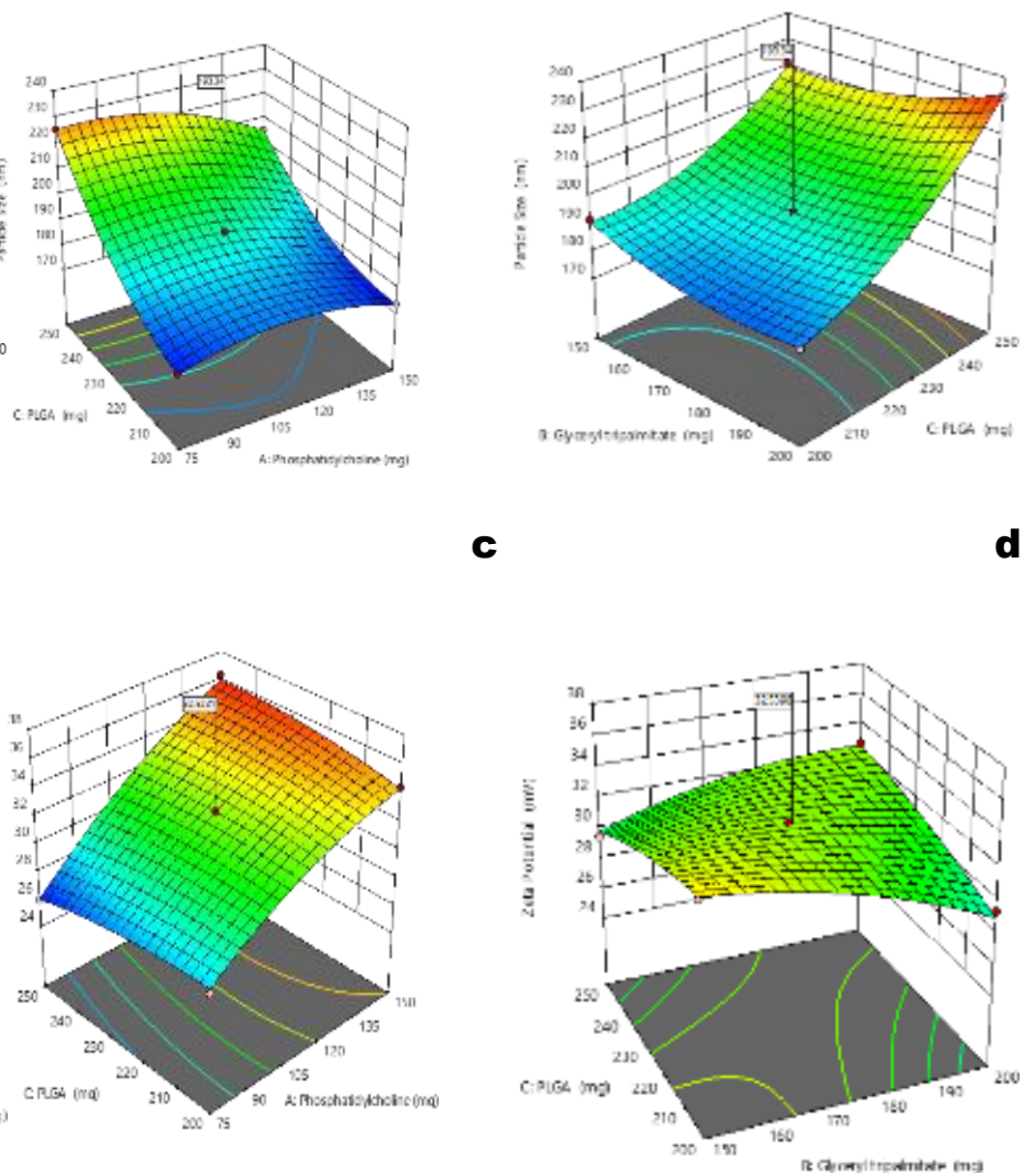
a

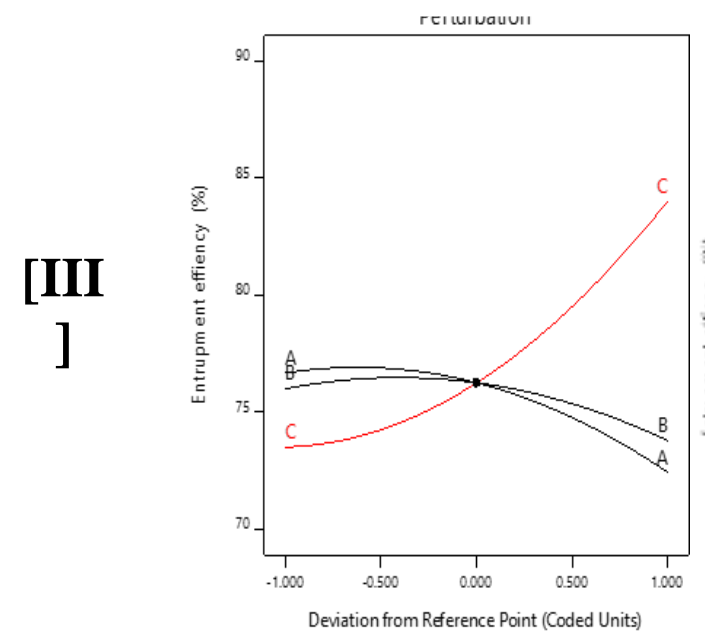

b

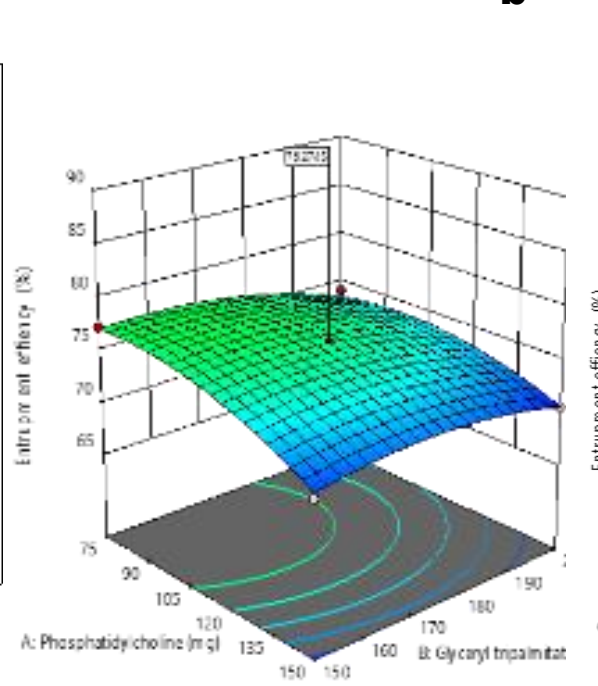

c

d
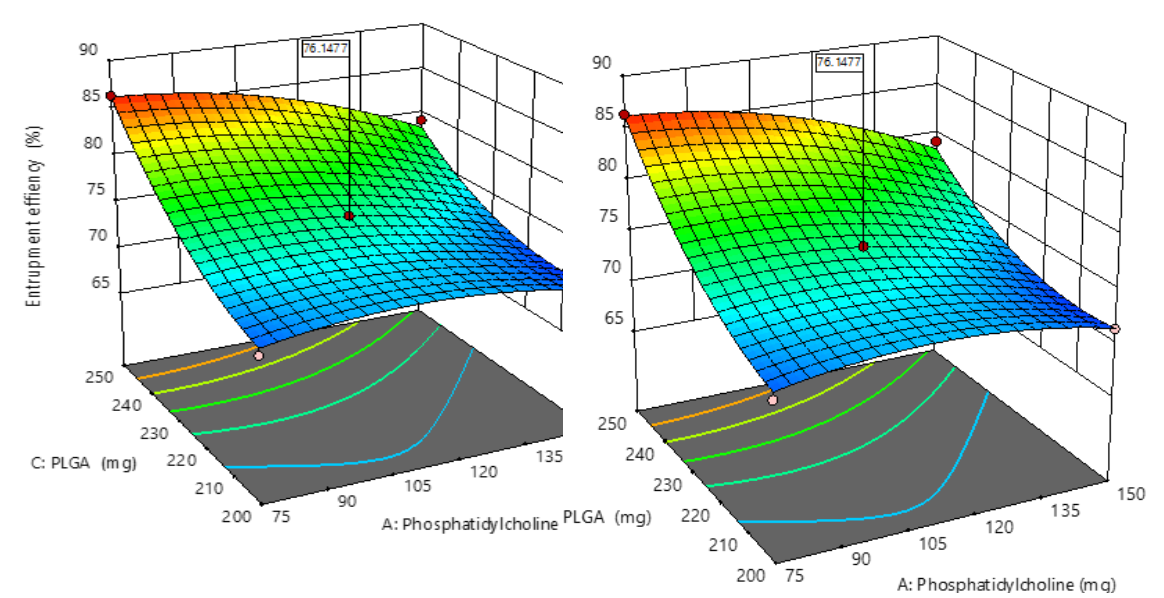
a

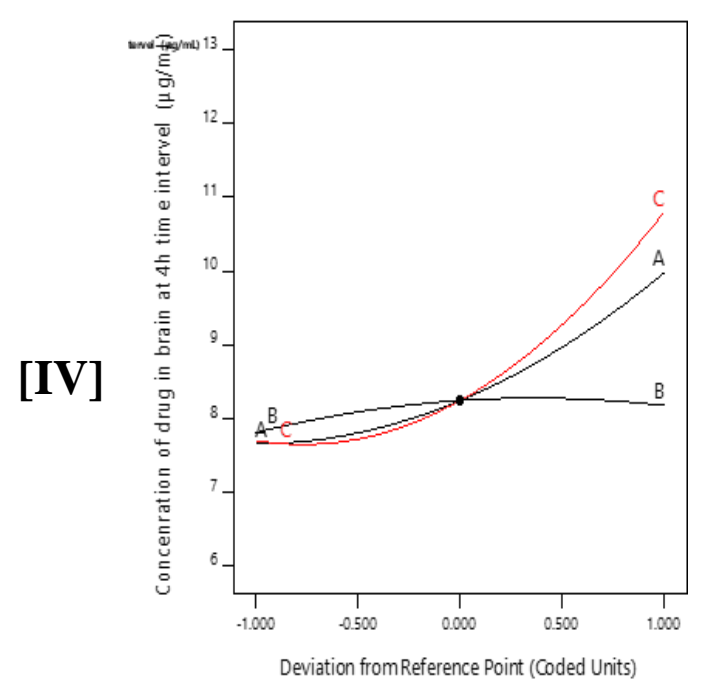

Deviation from Reference Point (Coded Units) b

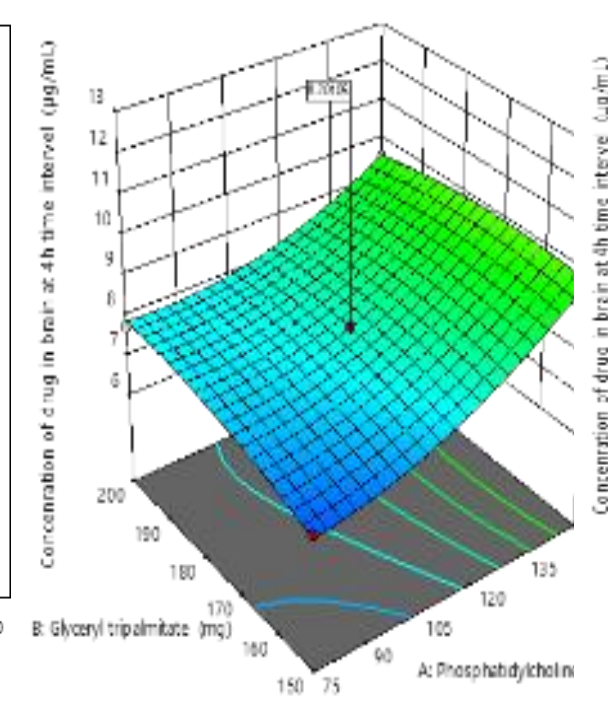

c

d

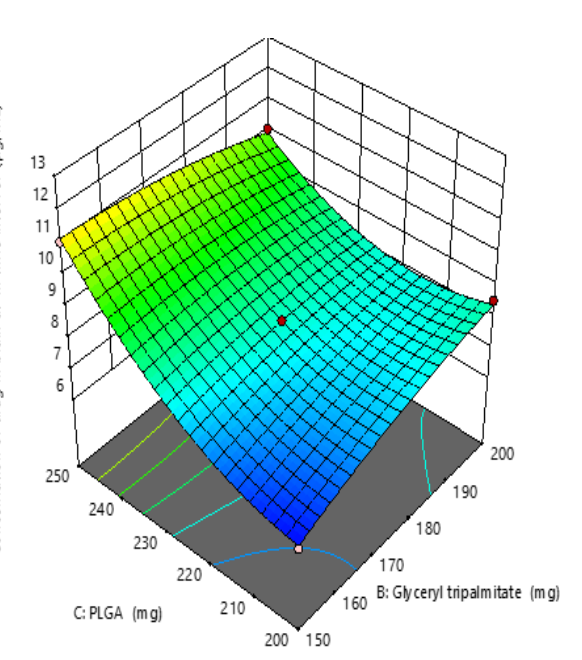

\section{Preprints (w ww.preprints.org) | NOT PEER-REVIEWED | Posted: 21 July 2021}

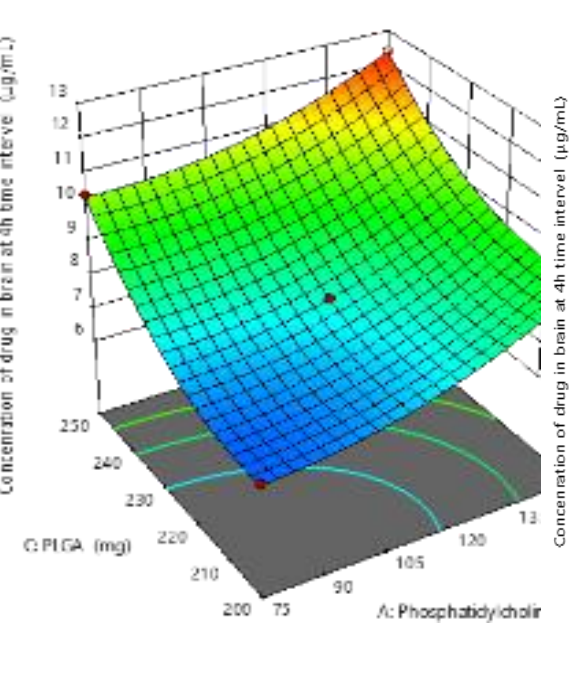

C: PLGA (mg)

.


[V]

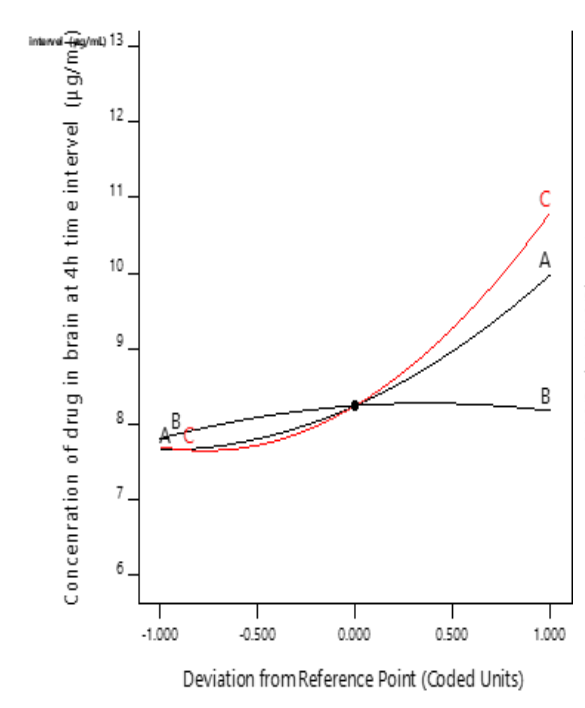

b

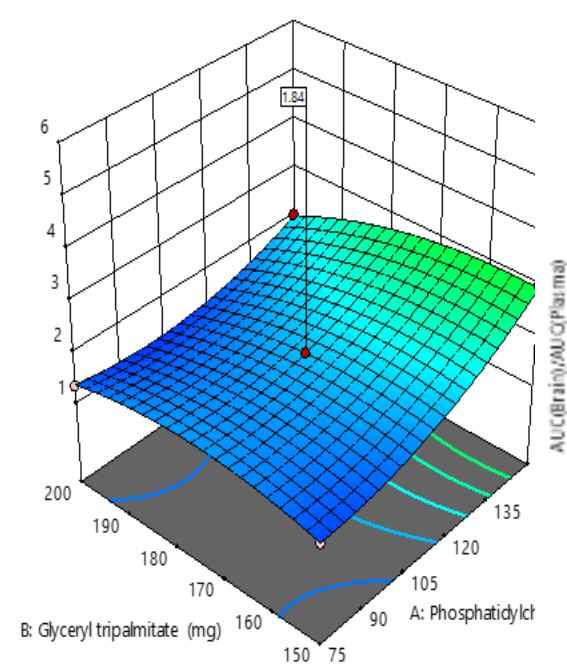

Fig.3: [I] Effects of particle size Particle Size(Y1) on Perturbation (a)., Effects of particle size Particle Size(Y1) on Glyceryl tripalmitate \& Phosphatidylcholine (b)., Effects of particle size Particle Size(Y1) on PLGA \& Phosphatidylcholine (c)., Effects of particle size Particle Size(Y1) on Glyceryl tripalmitate \& PLGA. [II] Effects of Zeta potential (Y2) on Perturbation (a)., Effects of Zeta potential (Y2) on Glyceryl tripalmitate \& Phosphatidylcholine (b)., Effects of Zeta potential (Y2) on PL GA \& Phosphatidylcholine (c)., Effects of Zeta potential (Y2) on Glyceryl tripalmitate \& PLGA(d). [III] Effects of Entrapment efficacy (Y3) on Perturbation (a)., Effects of Entrapment efficacy (Y3) on Glyceryl tripalmitate \& Phosphatidylcholine (b)., Effects of Entrapment efficacy (Y3) on PL GA \& Phosphatidylcholine (c)., Effects of Entrapment efficacy (Y3) on Glyceryl tripalmitate \& PLGA. [IV] Effects of Concentration of drug in brain at 4 h interval(Y4) on Perturbation (a)., Effects of Concentration of drug in brain at $4 \mathrm{~h}$ interval(Y4) on Glyceryl tripalmitate \& Phosphatidylcholine (b)., Effects of Concentration of drug in brain at 4h interval(Y4) on PL GA \& Phosphatidylcholine (c)., Effects of Concentration of drug in brain at $4 \mathrm{~h}$ interval(Y4) on Glyceryl tripalmitate \& PLGA. [V]. Effects of AUC(Brain)/AUC(Plasma)(Y5) on Perturbation (a)., Effects of AUC(Brain)/AUC(Plasma)(Y5) on Glyceryl tripalmitate \& Phosphatidylcholine (b)., Effects of AUC(Brain)/AUC(Plasma)(Y5) on PL GA \& Phosphatidylcholine (c)., Effects of AUC(Brain)/AUC(Plasma) (Y5)on Glyceryl tripalmitate \& PLGA 


\section{8}
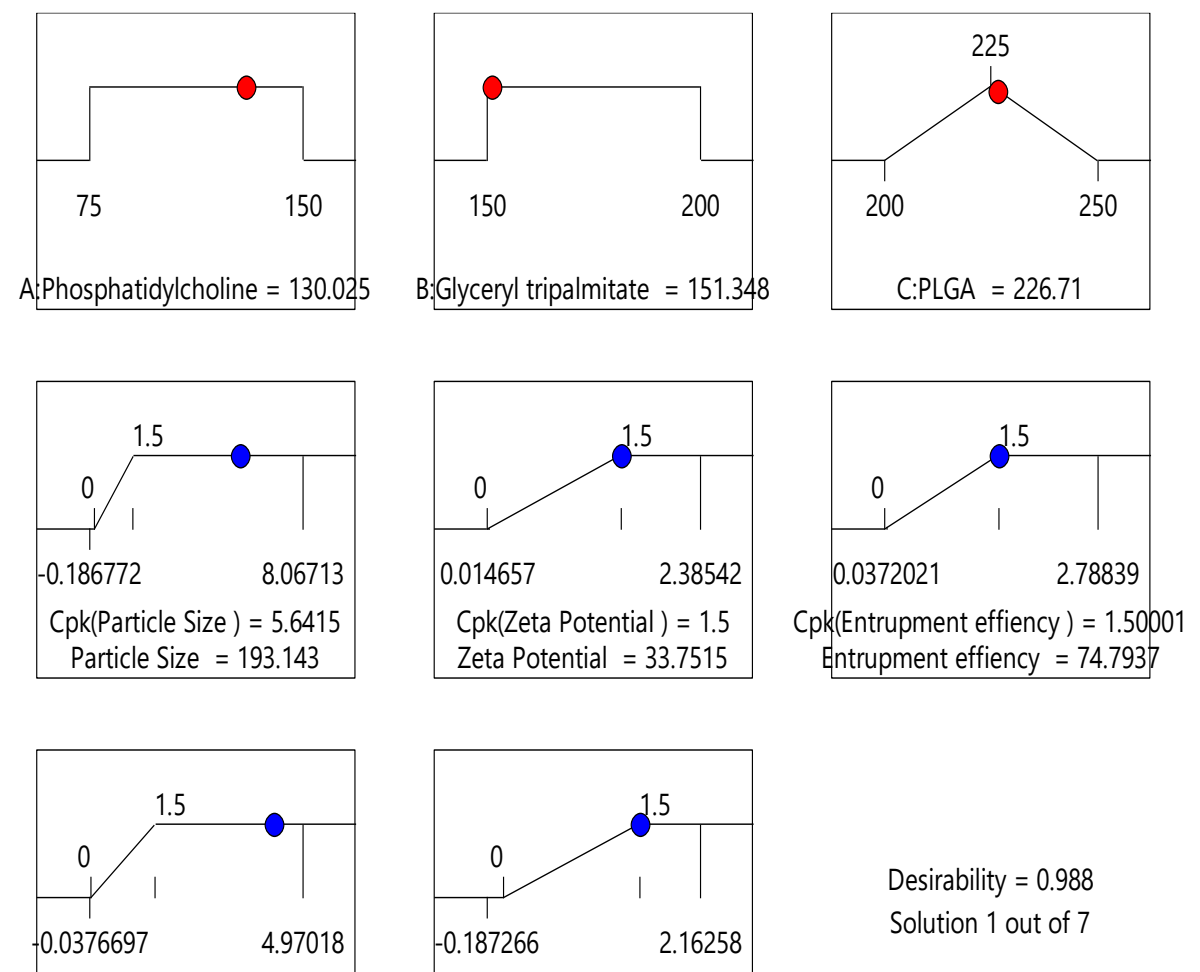

Cpk(Concenration of drug in brain at th time intervedpk(AUC(Brain)/AUC(Plasma)) $=1.5$

Concenration of drug in brain at $4 \mathrm{~h}$ time intervel AfC(Brain)/AUC(Plasma) $=2.37169$
Desirability $=0.988$ Solution 1 out of 7

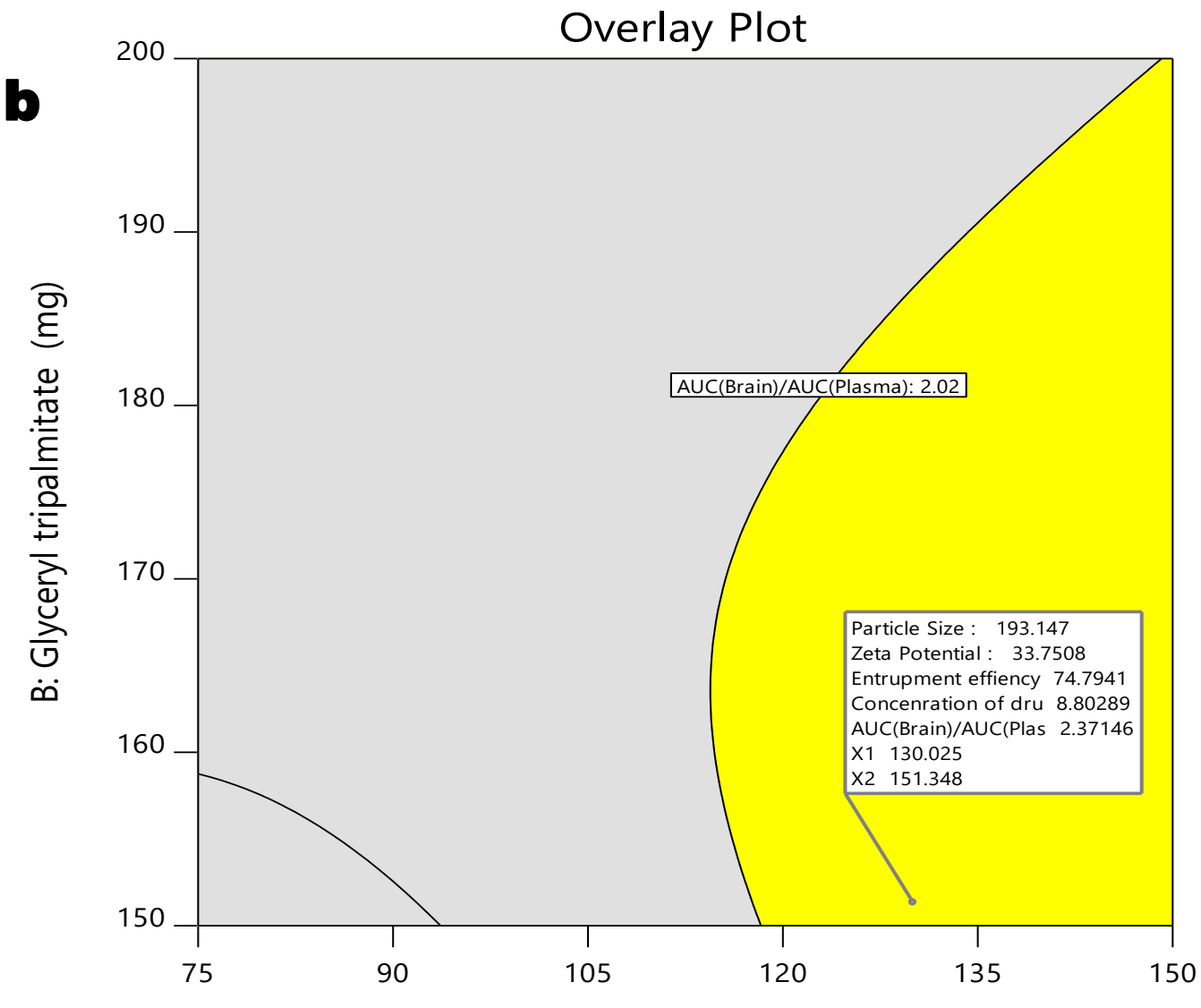

A: Phosphatidylcholine (mg)

Fig.4: Process optimization by Desirability approach (a) \& Overlay plot of design space and optimum batch (b) \\ Preprints (uruw.preprints.org) | NOT PEER-REVIEWED | Posted: 21 July 2021 \\ doi:10.20944/preprints202107.0503.v1}

Fig.4: Process optimization by Desirability approach (a) \& Overlay plot of design space and optimum batch (b) 
C

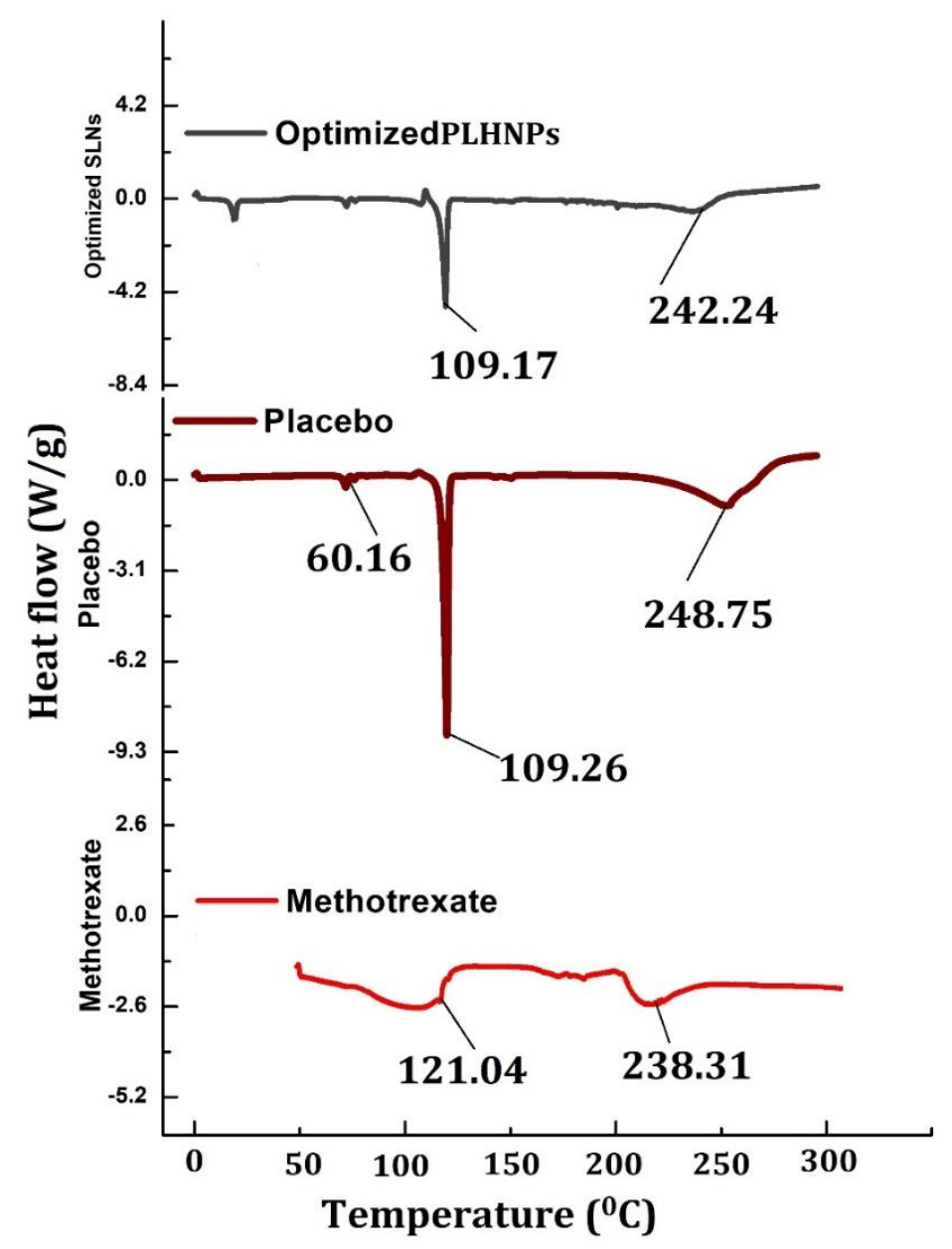

d

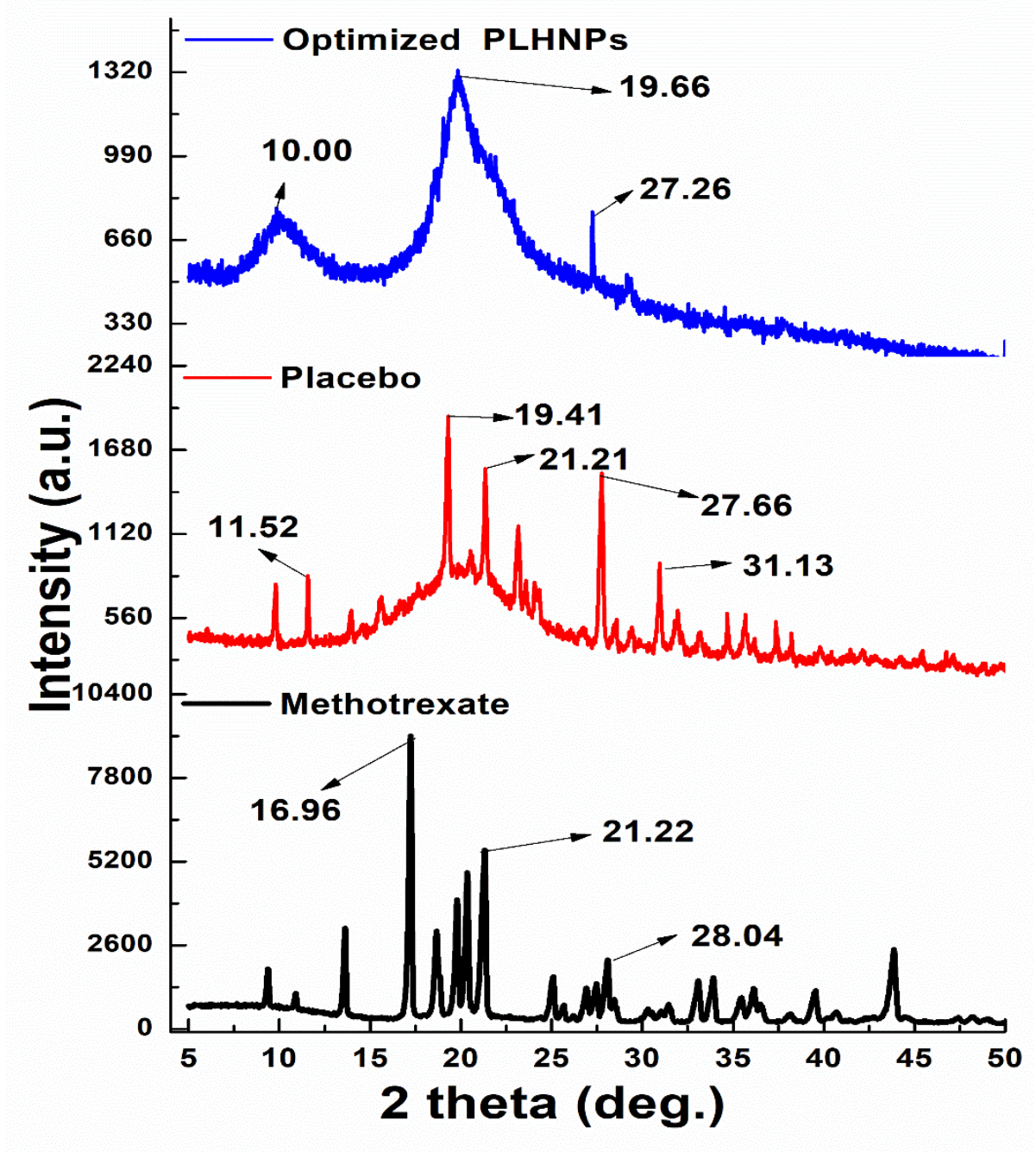




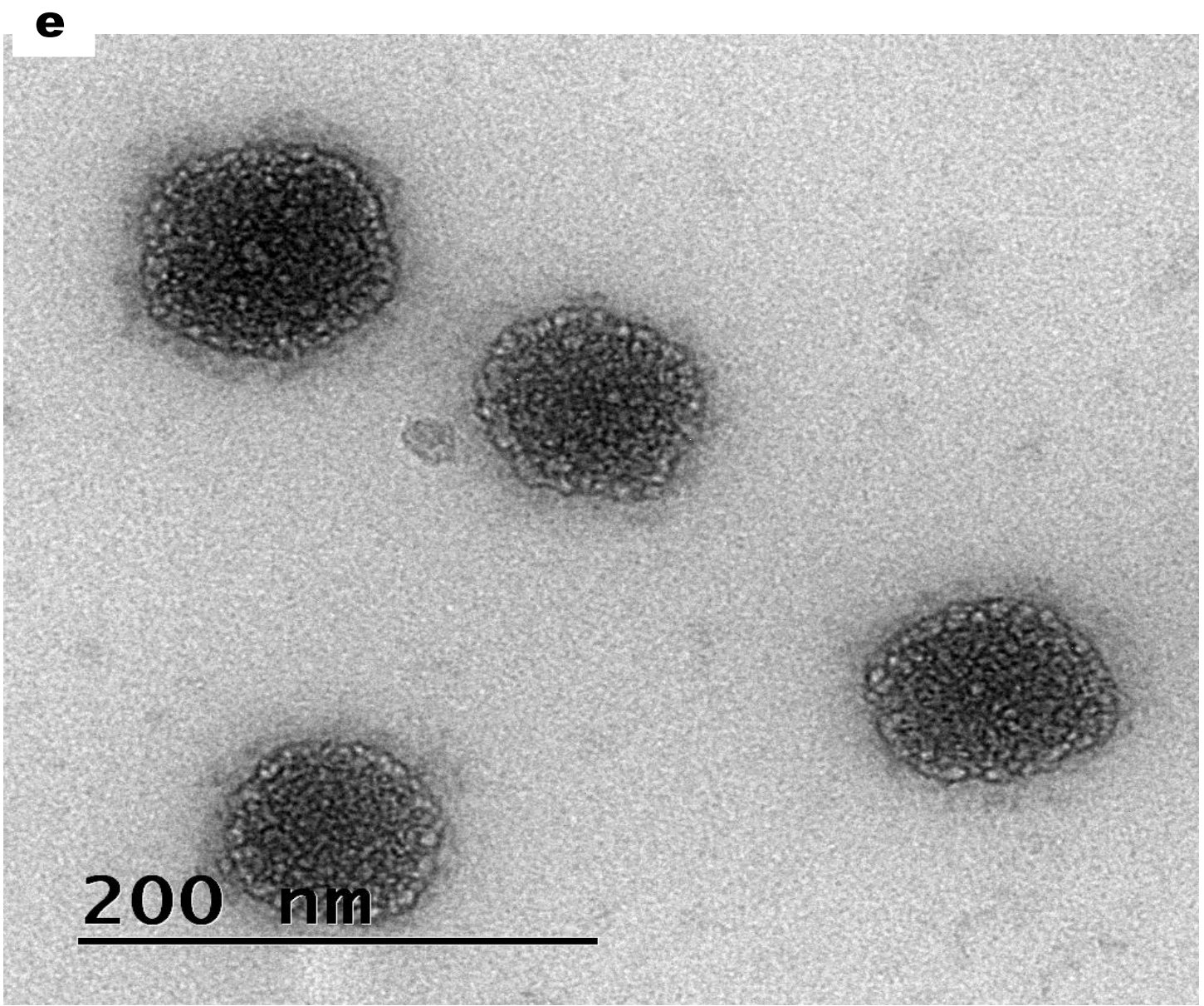

Fig.5: (a)in-vitro drug release profile of Methotrexate loaded PLGA lipid hybrid nanoparticles. (b). Fourier transform infrared spectroscopy of Methotrexate, PLGA, Placebo, Physical mixture and Optimized lipid hybrid nanoparticles. (c). DSC Thermograms of Methotrexate, Placebo and Optimized lipid hybrid nanoparticles. (d). XRD patterns of Methotrexate, Placebo and Optimized lipid hybrid nanoparticles. (e). Transmission electron microscopy of optimized Methotrexate loaded PLGA lipid hybrid nanoparticles 


\section{b}

a.

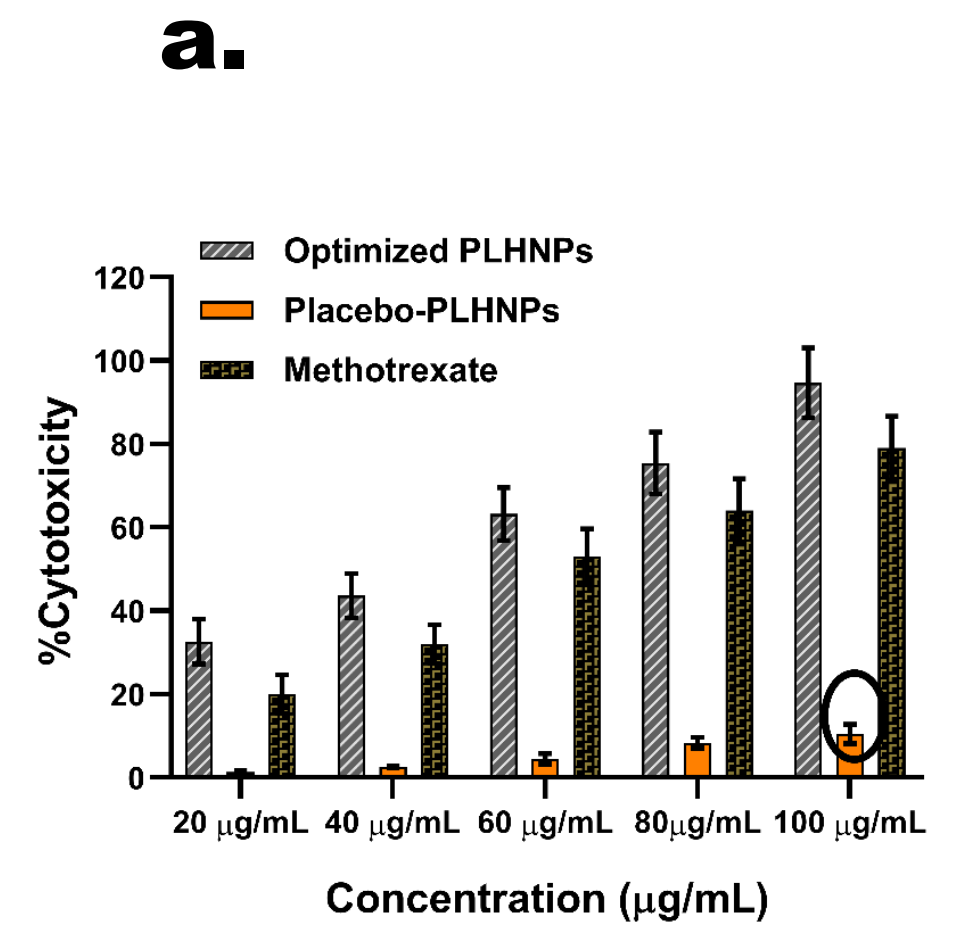

Fig.6: (a) in-vitro cytotoxicity of Methotrexate, Placebo- lipid hybrid nanoparticles and Optimized lipid hybrid nanoparticles. Values are represented as mean \pm SD $(n=3) \quad P<0.05$ (b) By using confocal laser scanning microscopy (CLSM) the cellular internalization of Optimized lipid hybrid nanoparticles in U-87 MG glioma cell lines were evaluated; where 4',6-diamidino-2phenylindole (DAPI) channel was used to stain nucleus

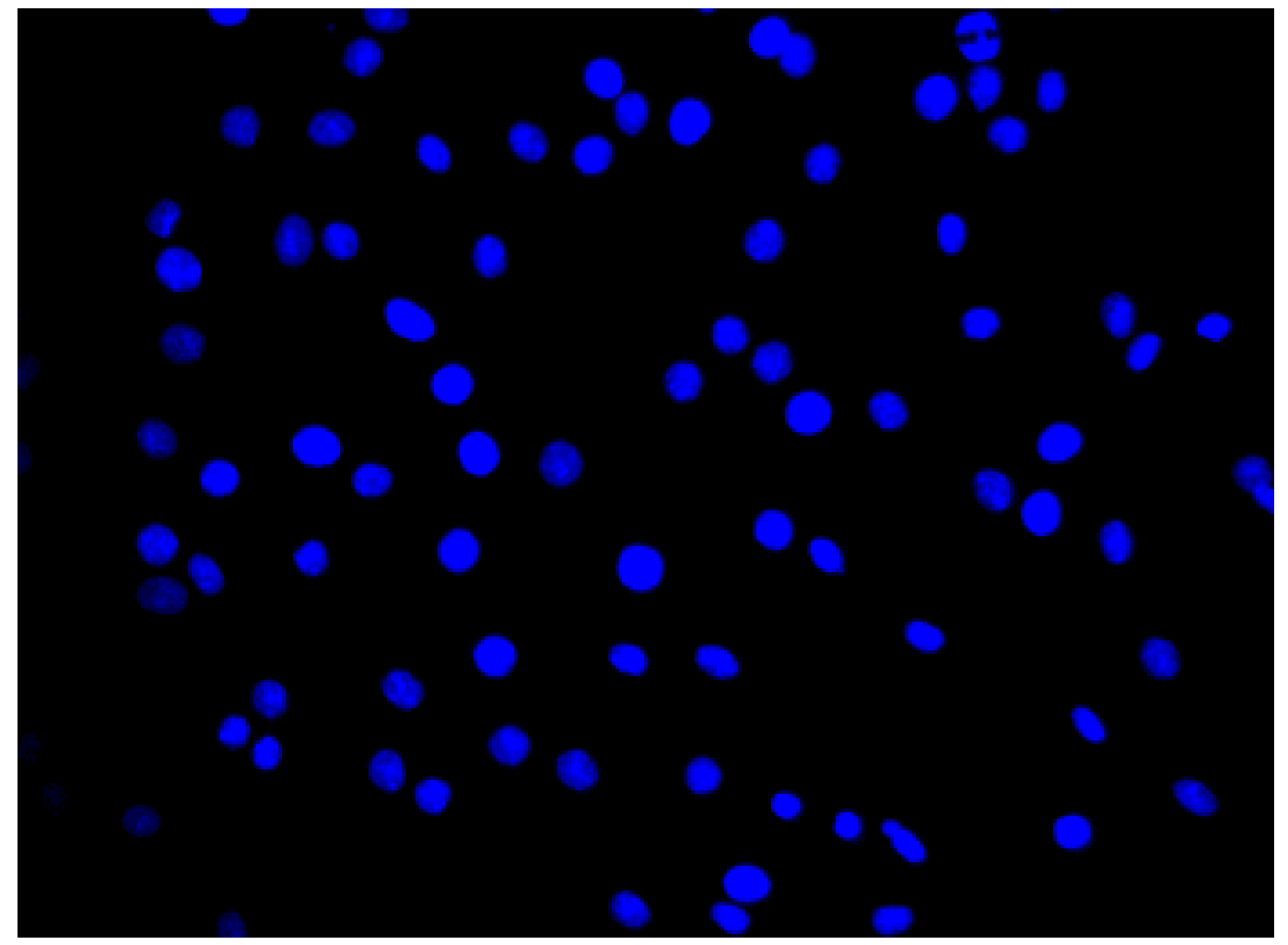

.

phentide (DAPI) chant was used to stain nucleus 


\section{(a) $10 \mu \mathrm{g} / \mathrm{mL}$}

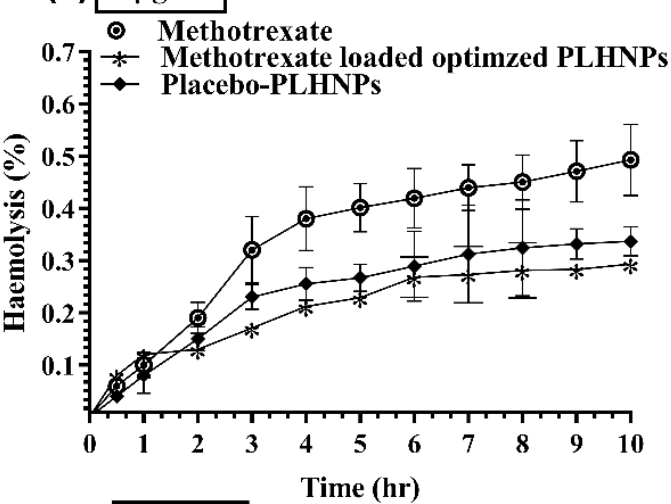

(b) $50 \mu \mathrm{g} / \mathrm{mL}$

$0.67 \begin{array}{ll}\odot & \text { Methotrexate } \\ * & \text { Methotrexate loaded optimzed PLHNPs }\end{array}$

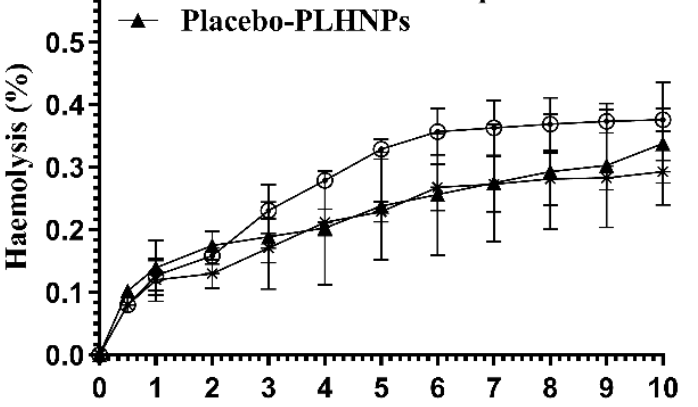

(c) $100 \mu \mathrm{g} / \mathrm{mL}$ Time (hr)

$\odot$ Methotrexate

$0.57^{*}$ - Methotrexate loaded optimzed PLHNPs

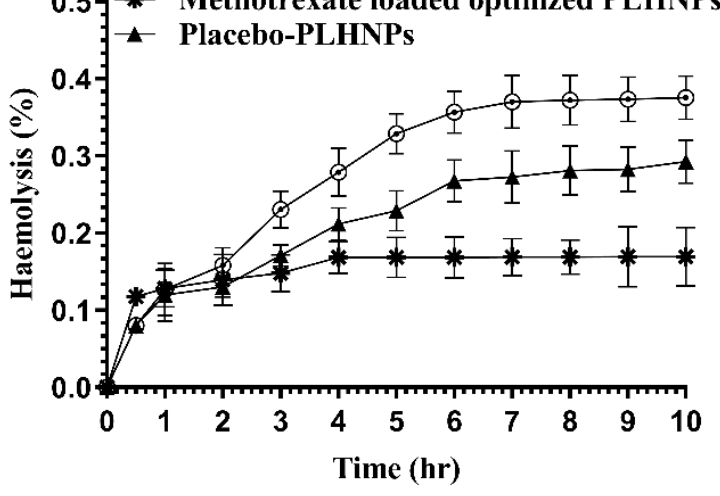

b

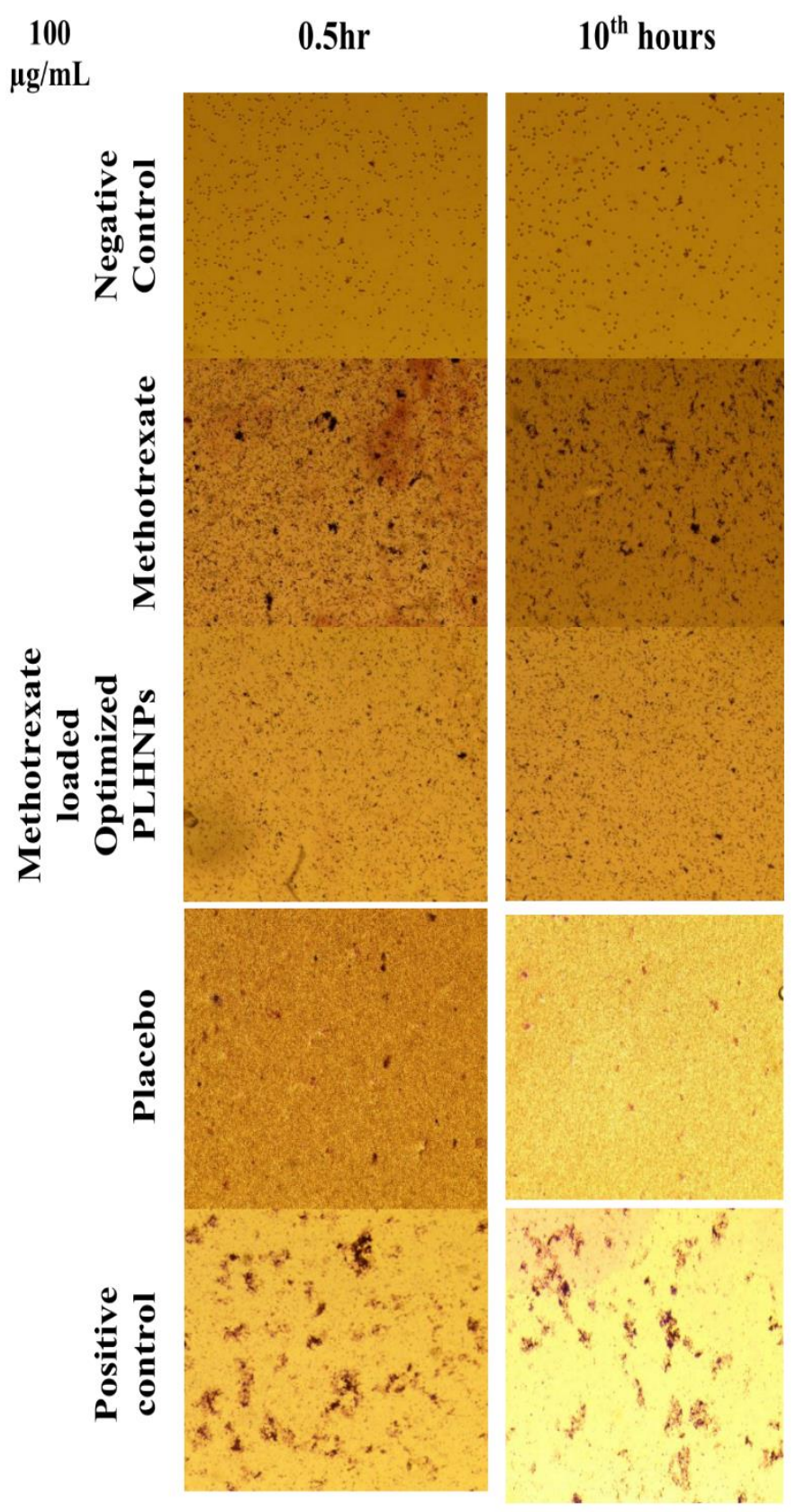

Fig.7: (a) Haemolysis (\%) profile of Methotrexate, Methotrexate loaded optimized lipid hybrid nanoparticles, Placebo-lipid hybrid nanoparticles after $10 \mu \mathrm{g} / \mathrm{mL}$ treatment; (b) Haemolysis (\%) profile of Methotrexate, Methotrexate loaded optimized lipid hybrid nanoparticles , Placebo-lipid hybrid nanoparticles after $50 \mu \mathrm{g} / \mathrm{mL}$ treatment; (c) Haemolysis (\%) profile of Methotrexate, Methotrexate loaded optimized lipid hybrid nanoparticles, Placebo- lipid hybrid nanoparticles after $100 \mu \mathrm{g} / \mathrm{mL}$ treatment; (D) High contrast microscopic images of erythrocytes during haemolysis (\%) studies after $100 \mu \mathrm{g} / \mathrm{mL}$ treatment of Methotrexate loaded optimized lipid hybrid nanoparticles, Placebo- lipid hybrid nanoparticles during 0.5 th hour and 10th hour 
(a)

Erythrocyte membrane integrity at $1^{\text {st }} \mathrm{hr}$

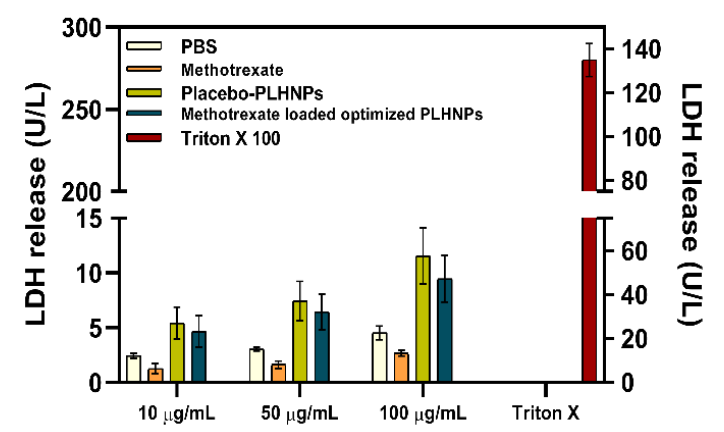

Test samples (b)

Erythrocyte membrane integrity at $4^{\text {th }} \mathrm{hr}$

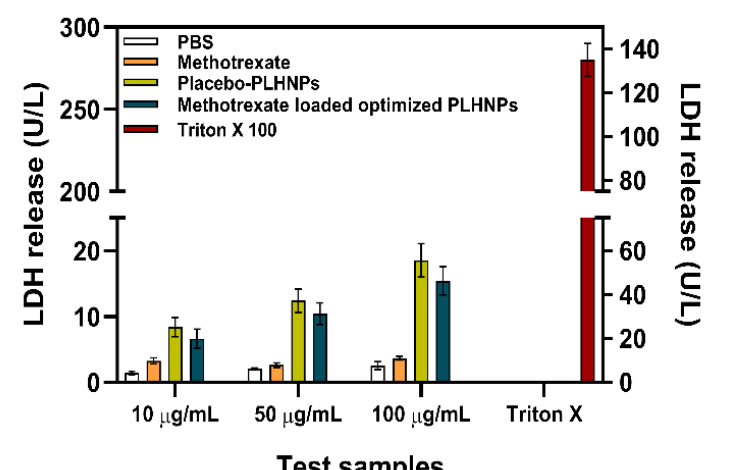

(c)

Erythrocyte membrane integrity at $8^{\text {th }} \mathrm{hr}$

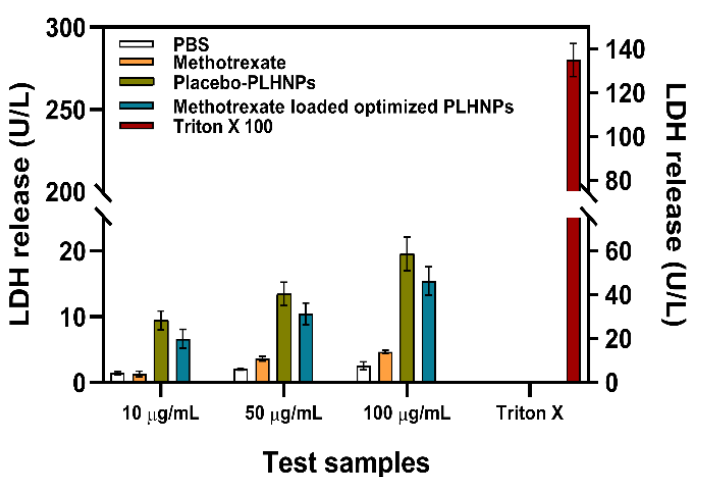

Fig.8: Amount of LDH release after treating with PBS, Methotrexate, Placebo- lipid hybrid nanoparticles and Methotrexate loaded optimized lipid hybrid nanoparticles at 1st hour (a), 4th hour(b) \& 8th hour (c) 


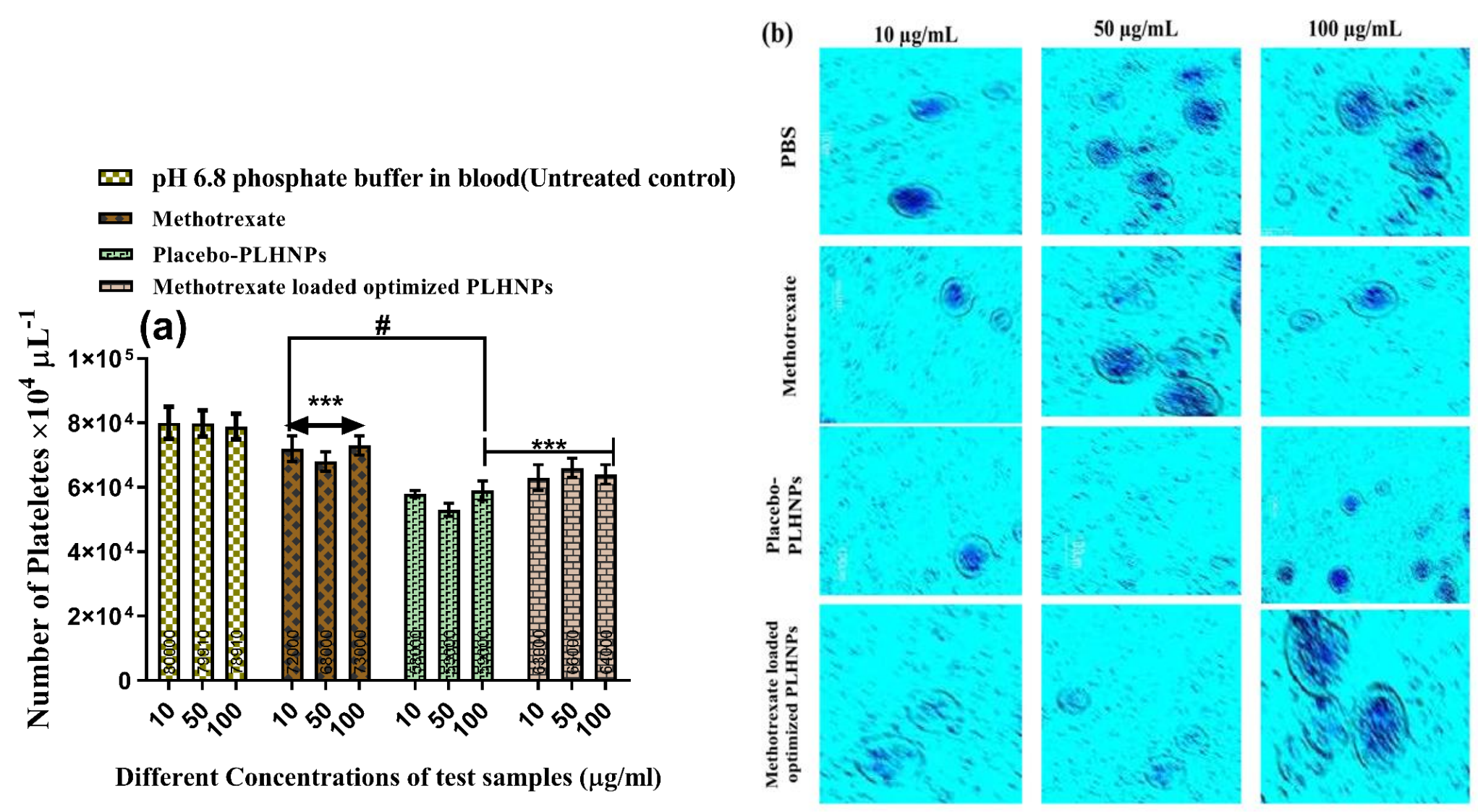

Fig.9: (a) Number of platelets counts after treatment with PBS, Methotrexate, Placebo- lipid hybrid nanoparticles and Methotrexate

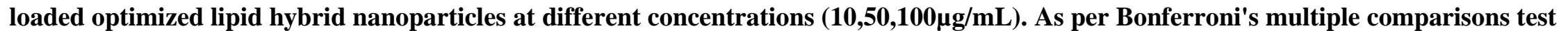
(values are represented as mean $\pm \mathrm{SD}(\mathrm{n}=3)$ ), *** indicates high statistical significance $(\mathrm{P}<0.001)$ and \# indicates insignificance $(\mathrm{P}=\mathbf{0 . 4 0 3 7})$ as compared to the different concentrations of Methotrexate; all the formulations were compared against with treated controlled group(Methotrexate Treatment). (b) Light microscopy images of plateletes with different treatments at prescribbed concnetration 


\author{
doi:10.20944/preprints202107.0503.v1
}
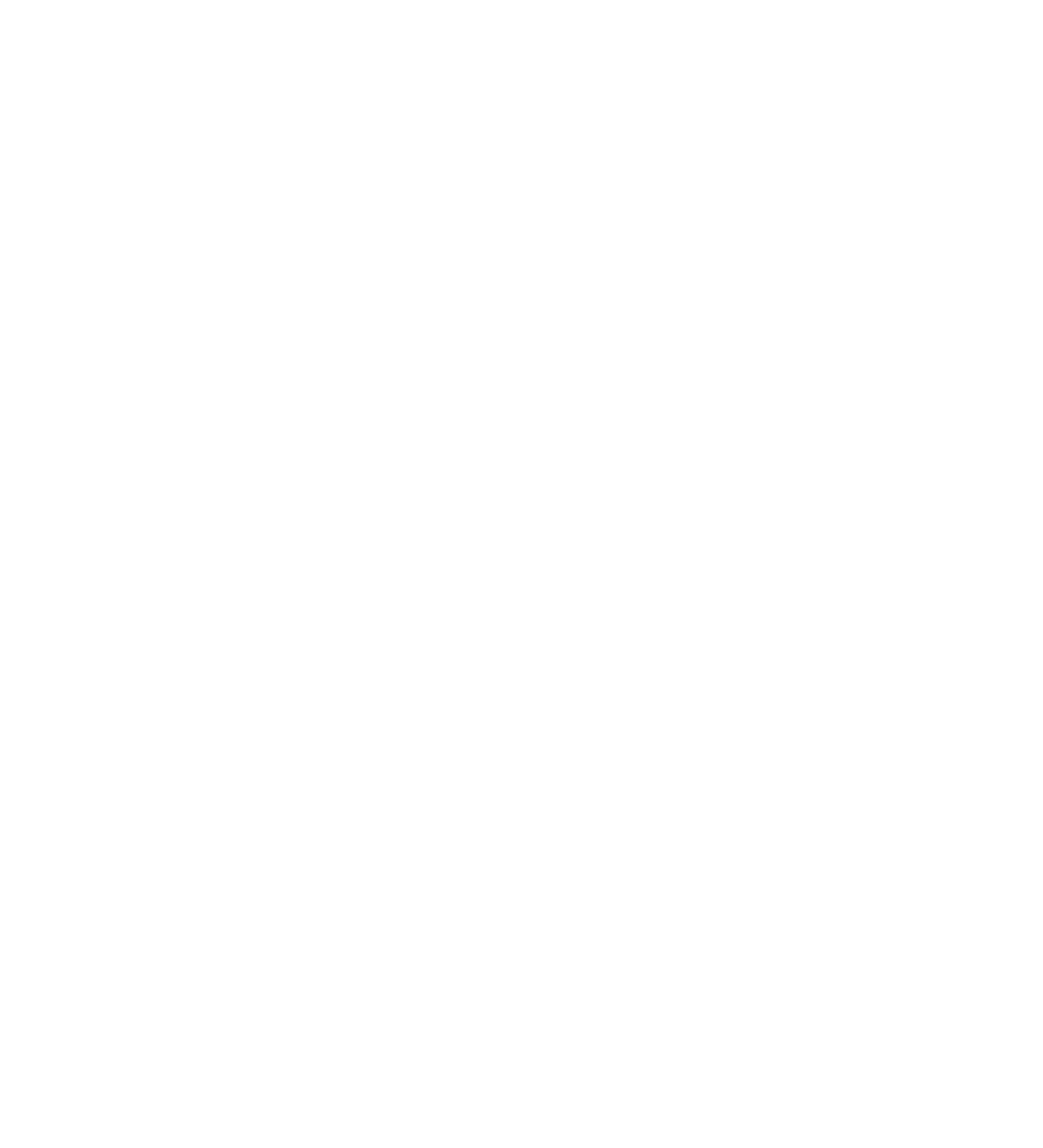